\title{
QUASILINEARIZATION FOR THE PERIODIC BOUNDARY VALUE PROBLEM FOR SYSTEMS OF IMPULSIVE DIFFERENTIAL EQUATIONS
}

\author{
S. G. HRISTOVA AND A. S. VATSALA
}

Received 20 June 2005; Revised 25 August 2005; Accepted 26 August 2005

The method of generalized quasilinearization for the system of nonlinear impulsive differential equations with periodic boundary conditions is studied. As a byproduct, the result for the system without impulses can be obtained, which is a new result as well.

Copyright (c) 2006 S. G. Hristova and A. S. Vatsala. This is an open access article distributed under the Creative Commons Attribution License, which permits unrestricted use, distribution, and reproduction in any medium, provided the original work is properly cited.

\section{Introduction}

The method of quasilinearization of Bellman and Kalaba [2] has been extended, refined, and generalized when the forcing function is the sum of a convex and concave function using coupled lower and upper solutions. This method is now known as the method of generalized quasilinearization. It has all the advantages of the quasilinearization method such that the iterates are solutions of linear systems and the sequences simultaneously converge to the unique solution of the nonlinear problem. See [1-4, 6-8] for details.

In this paper, we extend the method of generalized quasilinearization to system of nonlinear impulsive differential equations with periodic boundary conditions. For this purpose, we develop a linear comparison theorem for system of impulsive differential equations with periodic boundary conditions. We develop two iterates which are solutions of linear impulsive system with periodic boundary conditions which converge monotonically and quadratically to the unique solution of the nonlinear problem. Results related to different types of coupled lower and upper solutions are developed.We note that the results of [1] are a special case of our results where the forcing function is made to be convex and in addition they obtain semiquadratic convergence only. The results of [3] can be obtained as the scalar case of our result.

\section{Preliminary notes and definitions}

Let the points $\tau_{k} \in(0, T)$ be fixed such that $\tau_{k+1}>\tau_{k}, k=1,2, \ldots, p, \tau_{0}=0, \tau_{p+1}=T$. 
Consider the system of nonlinear impulsive differential equations (PBVP)

$$
\begin{gathered}
x^{\prime}=f(t, x(t))+g(t, x(t)) \quad \text { for } t \in[0, T], t \neq \tau_{k}, \\
x\left(\tau_{k}+0\right)=I_{k}\left(x\left(\tau_{k}\right)\right)+G_{k}\left(x\left(\tau_{k}\right)\right),
\end{gathered}
$$

with periodic boundary conditions

$$
x(0)=x(T)
$$

where $x \in \mathbb{R}^{n}, f, g:[0, T] \times \mathbb{R}^{n} \rightarrow \mathbb{R}^{n}, I_{k}, G_{k}: \mathbb{R}^{n} \rightarrow \mathbb{R}^{n}(k=1,2, \ldots, p)$.

We consider the set $P C(X, Y)$ of all functions $u: X \rightarrow Y,\left(X, Y \subset \mathbb{R}^{n}\right)$ which are piecewise continuous in $X$ with points of discontinuity of first kind at the points $\tau_{k} \in X$, that is, there exist the $\operatorname{limits}_{\lim _{t \downarrow} \tau_{k}} u(t)=u\left(\tau_{k}+0\right)<\infty$ and $\lim _{t \uparrow \tau_{k}} u(t)=u\left(\tau_{k}-0\right)=u\left(\tau_{k}\right)$.

We consider the set $P C^{1}(X, Y)$ of all functions $u \in P C(X, Y)$ that are continuously differentiable for $t \in X, t \neq \tau_{k}$.

Let the functions $\alpha, \beta \in P C\left([0, T], \mathbb{R}^{n}\right)$ be such that $\alpha(t) \leq \beta(t)$.

Consider the sets

$$
\begin{gathered}
S(\alpha, \beta)=\left\{u \in P C\left([0, T], \mathbb{R}^{n}\right): \alpha(t) \leq u(t) \leq \beta(t) \text { for } t \in[0, T]\right\}, \\
\Omega(\alpha, \beta)=\left\{(t, x) \in[0, T] \times \mathbb{R}^{n}: \alpha(t) \leq x \leq \beta(t)\right\}, \\
D_{k}(\alpha, \beta)=\left\{x \in \mathbb{R}^{n}: \alpha\left(\tau_{k}\right) \leq x \leq \beta\left(\tau_{k}\right)\right\}, \quad k=1,2, \ldots, p .
\end{gathered}
$$

Definition 2.1. The function $\alpha_{0}(t) \in P C^{1}\left([0, T], \mathbb{R}^{n}\right)$ is a lower solution of PBVP (2.1)(2.2) if

$$
\begin{gathered}
\alpha_{0}^{\prime}(t) \leq f\left(t, \alpha_{0}(t)\right)+g\left(t, \alpha_{0}(t)\right) \quad \text { for } t \in[0, T], t \neq \tau_{k} \\
\alpha_{0}\left(\tau_{k}+0\right) \leq I_{k}\left(\alpha_{0}\left(\tau_{k}\right)\right)+G_{k}\left(\alpha_{0}\left(\tau_{k}\right)\right) \\
\alpha_{0}(0) \leq \alpha_{0}(T) .
\end{gathered}
$$

If the inequalities are reversed, then $\alpha_{0}(t)$ is called an upper solution. This is referred to as natural upper and lower solutions.

Definition 2.2. The functions $\alpha_{0}(t), \beta_{0}(t) \in P C^{1}\left([0, T], \mathbb{R}^{n}\right)$ are coupled lower and upper solutions of PBVP (2.1)-(2.2) of type I if

$$
\begin{gathered}
\alpha_{0}^{\prime}(t) \leq f\left(t, \alpha_{0}(t)\right)+g\left(t, \beta_{0}(t)\right), \\
\beta_{0}^{\prime}(t) \geq f\left(t, \beta_{0}(t)\right)+g\left(t, \alpha_{0}(t)\right) \quad \text { for } t \in[0, T], t \neq \tau_{k}, \\
\alpha_{0}\left(\tau_{k}+0\right) \leq I_{k}\left(\alpha_{0}\left(\tau_{k}\right)\right)+G_{k}\left(\beta_{0}\left(\tau_{k}\right)\right), \\
\beta_{0}\left(\tau_{k}+0\right) \geq I_{k}\left(\beta_{0}\left(\tau_{k}\right)\right)+G_{k}\left(\alpha_{0}\left(\tau_{k}\right)\right), \\
\alpha_{0}(0) \leq \alpha_{0}(T), \quad \beta_{0}(0) \geq \beta_{0}(T) .
\end{gathered}
$$


Definition 2.3. The functions $\alpha_{0}(t), \beta_{0}(t) \in P C^{1}\left([0, T], \mathbb{R}^{n}\right)$ are coupled lower and upper solutions of PBVP (2.1)-(2.2) of type II if

$$
\begin{gathered}
\alpha_{0}^{\prime}(t) \leq f\left(t, \beta_{0}(t)\right)+g\left(t, \alpha_{0}(t)\right), \\
\beta_{0}^{\prime}(t) \geq f\left(t, \alpha_{0}(t)\right)+g\left(t, \beta_{0}(t)\right) \quad \text { for } t \in[0, T], t \neq \tau_{k}, \\
\alpha_{0}\left(\tau_{k}+0\right) \leq I_{k}\left(\beta_{0}\left(\tau_{k}\right)\right)+G_{k}\left(\alpha_{0}\left(\tau_{k}\right)\right), \\
\beta_{0}\left(\tau_{k}+0\right) \geq I_{k}\left(\alpha_{0}\left(\tau_{k}\right)\right)+G_{k}\left(\beta_{0}\left(\tau_{k}\right)\right), \\
\alpha_{0}(0) \leq \alpha_{0}(T), \quad \beta_{0}(0) \geq \beta_{0}(T) .
\end{gathered}
$$

One can define other kinds of coupled lower and upper solutions of (2.1)-(2.2) on the same lines.

We will prove some preliminary results for linear systems of impulsive differential equations.

Let $A=\left\{a_{i j}\right\}_{i, j=1}^{N}$ be a matrix, $N$ a natural number. We will say that $A>0$ if $a_{i j}>0$ for $i, j=1,2, \ldots, N$.

Definition 2.4. The matrix $B=\left\{b_{i j}\right\}_{i, j=1}^{N}$ belongs to the class $\Psi$ if

(P1) $B \geq 0$;

(P2) for $i: 1 \leq i \leq N: \sum_{j=1}^{N} b_{i j} \leq 1$.

Definition 2.5. The matrix $A(t)=\left\{a_{i j}(t)\right\}_{i, j=1}^{N}$ belongs to the class $\Xi$ if

(P1) $a_{i j}(t) \in C([0, T], \mathbb{R}), a_{i j}(t) \geq 0$ for $j \neq i, i, j=1,2, \ldots, N, t \in[0, T]$;

(P2) $\sum_{j \neq i} a_{i j}(t)+a_{i i}(t)<0, t \in[0, T], i=1,2, \ldots, N$.

We will define the following operation between vectors: let $x=\left(x_{1}, x_{2}, \ldots, x_{N}\right), y=$ $\left(y_{1}, y_{2}, \ldots, y_{N}\right)$; then $x @ y=\left(x_{1} y_{1}, x_{2} y_{2}, \ldots, x_{N} y_{N}\right)$.

We will use the following notation: $e=(1,1, \ldots, 1)$. We will note that the vector $e$ is the unit vector according to the operation @.

For our main results, we need the following lemma for linear systems of impulsive differential inequalities.

Lemma 2.6. Assume that

(1) the matrix $A(t)=\left\{a_{i j}(t)\right\}_{i, j=1}^{N}$ belongs to the class $\Xi$;

(2) the matrices $B_{k}=\left\{b_{i j}^{(k)}\right\}_{i, j=1}^{N}, k=1, \ldots, p$, belong to the class $\Psi$;

(3) the function $m \in P C^{1}\left([0, T], \mathbb{R}^{N}\right)$ satisfies the inequalities

$$
\begin{gathered}
m^{\prime}(t) \leq A(t) m(t), \quad t \in[0, T], t \neq \tau_{k} \\
m\left(\tau_{k}+0\right) \leq B_{k} m\left(\tau_{k}\right), \\
m(0) \leq m(T) .
\end{gathered}
$$

Then $m(t) \leq 0$ for $t \in[0, T]$.

Proof. Let $\tau_{0}=0, \tau_{p+1}=T$. Consider the numbers

$$
\epsilon_{k+1}=\max _{1 \leq i \leq N} \sup _{t \in\left(\tau_{k}+0, \tau_{k+1}\right]} m_{i}(t), \quad k=0,1,2, \ldots, p .
$$


Case 1. Let $\epsilon_{k}>0$ for $k=1,2, \ldots, p+1$.

Consider the case when there exists a number $k: 0 \leq k \leq p$ such that $m_{j_{k}}\left(\xi_{k}\right)=\epsilon_{k+1}$ for some natural number $j_{k}$ and a point $\xi_{k} \in\left(\tau_{k}+0, \tau_{k+1}\right]$. Then $m_{j_{k}}^{\prime}\left(\xi_{k}\right)=\lim _{h \rightarrow 0+}\left(\left(m_{j_{k}}\left(\xi_{k}-\right.\right.\right.$ $\left.\left.h)-m_{j_{k}}\left(\xi_{k}\right)\right) /-h\right) \geq 0$ and from the inequality (2.7) we obtain

$$
0 \leq m_{j_{k}}^{\prime}\left(\xi_{k}\right) \leq \sum_{l \neq j_{k}} a_{j_{k} l}\left(\xi_{k}\right) m_{l}\left(\xi_{k}\right)+a_{j_{k} j_{k}}\left(\xi_{k}\right) m_{j_{k}}\left(\xi_{k}\right) \leq\left(\sum_{l \neq j_{k}} a_{j_{k} l}\left(\xi_{k}\right)+a_{j_{k} j_{k}}\left(\xi_{k}\right)\right) \epsilon_{k+1}<0
$$

The obtained contradiction proves that this case is not possible.

Consider the case when for every $k: 0 \leq k \leq p$ there exists a natural number $j_{k}$ such that

$$
\lim _{t \rightarrow \tau_{k}+0} m_{j_{k}}(t)=\epsilon_{k+1}
$$

and $m_{i}(t)<\epsilon_{k+1}$ for $t \in\left(\tau_{k}, \tau_{k+1}\right], i=1,2, \ldots, N$. Then from the jump condition (2.8) we have $\epsilon_{k+1}=m_{j_{k}}\left(\tau_{k}+0\right) \leq \sum_{i=1}^{N} b_{j_{k} i}^{(k)} m_{i}\left(\tau_{k}\right)<\left(\sum_{i=1}^{N} b_{j_{k} i}^{(k)}\right) \epsilon_{k} \leq \epsilon_{k}$. By induction, we obtain that $m_{j_{0}}(T)<\epsilon_{p+1} \leq \epsilon_{p} \leq \cdots \leq \epsilon_{1}=m_{j_{0}}(0)$. The last inequality contradicts the condition (2.9). Therefore, this case is impossible.

Case 2. There exists a natural number $l: 1 \leq l \leq p+1$ such that $\epsilon_{l} \leq 0$. Let $k=\max \{l$ : $\left.\epsilon_{l} \leq 0\right\}$.

If $k=p+1$, then $m(T) \leq 0$.

If $k<p+1$, then $\epsilon_{k+1}>0$. According to the jump condition (2.8), we obtain $m\left(\tau_{k}+\right.$ $0) \leq B_{k} m\left(\tau_{k}\right) \leq 0$. Therefore, there exist a natural number $j_{k}$ and a point $\xi_{k} \in\left(\tau_{k}, \tau_{k+1}\right]$ such that $m_{j_{k}}\left(\xi_{k}\right)=\epsilon_{k+1}$ and $m_{j_{k}}^{\prime}\left(\xi_{k}\right) \geq 0$. From the inequality (2.7), we have

$$
0 \leq m_{j_{k}}^{\prime}\left(\xi_{k}\right) \leq \sum_{l \neq j_{k}} a_{j_{k} l}\left(\xi_{k}\right) m_{l}\left(\xi_{k}\right)+a_{j_{k} j_{k}}\left(\xi_{k}\right) m_{j}\left(\xi_{k}\right) \leq\left(\sum_{l \neq j_{k}} a_{j_{k} l}\left(\xi_{k}\right)+a_{j_{k} j_{k}}\left(\xi_{k}\right)\right) \epsilon_{k+1}<0 .
$$

The obtained contradiction proves that $k=p+1$. Therefore, $m(T) \leq 0$ and from the boundary condition (2.9) it follows that $m(0) \leq 0$. As in the proof above, we obtain that $\epsilon_{l} \leq 0$ for $l=1,2, \ldots, p$. Therefore, $m(t) \leq 0$ on $[0, T]$.

As an application of Lemma 2.6, the following corollary is implied. This will be useful in proving the existence and uniqueness of the linear nonhomogeneous impulsive system with periodic boundary condition.

Corollary 2.7. Let conditions (1) and (2) of Lemma 2.6 be satisfied.

Then the PBVP for the homogeneous linear system

$$
\begin{gathered}
m^{\prime}(t)=A(t) m(t), \quad t \in[0, T], t \neq \tau_{k} \\
m\left(\tau_{k}+0\right)=B_{k} m\left(\tau_{k}\right) \\
m(0)=m(T)
\end{gathered}
$$

has only the trivial solution. 
We note that the solution of the linear system of impulsive equations (2.14), (2.15) with the initial condition $m(0)=m_{0}$ is $m(t)=W(t, 0) m_{0}$, where

$$
W(t, s)= \begin{cases}U_{k}(t, s) & \text { for } t, s \in\left(t_{k-1}, t_{k}\right], \\ U_{k+1}\left(t, t_{k}\right) B_{k} U_{k}\left(t_{k}, s\right) & \text { for } t_{k-1}<s \leq t_{k}<t \leq t_{k+1}, \\ U_{k+1}\left(t, t_{k}\right) \prod_{j=i+1}^{k} B_{j} U_{j}\left(t_{j}, t_{j-1}\right) B_{i} U_{i}\left(t_{i}, s\right) & \text { for } t_{i-1}<s \leq t_{i}<t_{k}<t \leq t_{k+1},\end{cases}
$$

and $U_{k}(t, s)$ is the fundamental matrix of the linear system $m^{\prime}=A(t) m(t), t \in\left(\tau_{k}, \tau_{k+1}\right]$ (for more details, see $[5,9]$ ).

Lemma 2.8. Let $A(t)=\left\{a_{i j}(t)\right\}_{i, j=1}^{N} \in \Xi$ and $B_{k}=\left\{b_{i j}^{(k)}\right\}_{i, j=1}^{N} \in \Psi, k=1, \ldots, p$.

Then $\operatorname{det}(E-W(t, 0)) \neq 0$, where $E$ is the unit $N \times N$ matrix.

Proof. If $m(0)=m(T)$, then $m_{0}=W(T, 0) m_{0}$ and $(I-W(T, 0)) m_{0}=0$. According to Corollary 2.7, the linear system (2.14)-(2.16) has only the trivial solution, so $m_{0}=0$ and therefore $\operatorname{det}(E-W(t, 0)) \neq 0$.

Consider the periodic boundary value problem for the nonhomogeneous linear systems of impulsive differential equations

$$
\begin{gathered}
m^{\prime}(t)=A(t) m(t)+h(t), \quad t \in[0, T], t \neq \tau_{k}, \\
m\left(\tau_{k}+0\right)=B_{k} m\left(\tau_{k}\right)+\sigma_{k}, \\
m(0)=m(T) .
\end{gathered}
$$

Lemma 2.9 (see [5, Theorem 2.5.1]). Assume that the matrix $(E-W(t, 0))$ is nonsingular and the function $h \in P C^{1}\left([0, T], \mathbb{R}^{N}\right)$.

Then the PBVP (2.18)-(2.20) has a unique solution $m(t)$ given by the formula

$$
m(t)=W(t, 0) m_{0}+\int_{0}^{t} W(t, s) h(s) d s+\sum_{0<t_{k}<t} W\left(t, t_{k}+0\right) \sigma_{k},
$$

where

$$
m_{0}=(E-W(T, 0))^{-1}\left(\int_{0}^{T} W(T, s) h(s) d s+\sum_{k=1}^{p} W\left(T, t_{k}+0\right) \sigma_{k}\right),
$$

and $W(t, s)$ is defined by (2.17).

We will need the following comparison result.

Lemma 2.10. Assume that

(1) the matrix $A(t)=\left\{a_{i j}(t)\right\}_{i, j=1}^{N}$ belongs to the class $\Xi$;

(2) the matrices $B_{k}, k=1, \ldots, p$, belong to the class $\Psi$ and $\sigma_{k}$ are constants;

(3) the function $h \in P C\left([0, T], \mathbb{R}^{N}\right)$;

(4) the functions $v(t)$ and $w(t)$ are lower and upper solutions of the periodic boundary value problem for the linear nonhomogeneous system (2.18)-(2.20). 
Then

$$
v(t) \leq W(t, 0) m_{0}+\int_{0}^{t} W(t, s) h(s) d s+\sum_{0<t_{k}<t} W\left(t, t_{k}+0\right) \sigma_{k} \leq w(t) \quad \text { for } t \in[0, T],
$$

where $m_{0}$ is given by (2.22), and $W(t, s)$ is defined by (2.17).

Proof. Consider the functions $p(t)=v(t)-x(t)$ and $q(t)=x(t)-w(t)$, where $x(t)$ is the solution of (2.17)-(2.19). Both functions satisfy the linear system of inequalities (2.7)(2.9) and according to Lemma 2.6 the functions are nonpositive on $[0, T]$, which proves Lemma 2.10.

In our main result, we will use the following integral mean-value theorem.

Lemma 2.11. Let the function $F \in C^{1}\left[D, \mathbb{R}^{N}\right]$, where $D \subset \mathbb{R}^{N}$ is a convex set. Then

$$
F(x)-F(y)=\left(\int_{0}^{1} F_{x}(\lambda x+(1-\lambda) y) d \lambda\right)(x-y)
$$

\section{Main results}

In this section, we develop the method of quasilinearization for the periodic boundary value problem for the system of nonlinear impulsive differential equations (2.1)-(2.2). We obtain two monotone sequences which are solutions of appropriately chosen linear impulsive differential systems with periodic boundary conditions. These monotone sequences converge quadratically to the unique solution of (2.1)-(2.2).

THEOREM 3.1. Let the following conditions hold.

(1) The functions $\alpha_{0}(t), \beta_{0}(t) \in P C^{1}\left([0, T], \mathbb{R}^{n}\right), \alpha_{0}(t) \leq \beta_{0}(t)$ for $t \in[0, T]$, are coupled lower and upper solutions of the PBVP (2.1)-(2.2) of type I.

(2) The functions $f_{x}, g_{x}$ exist and are continuous on $\Omega\left(\alpha_{0}, \beta_{0}\right), f_{x}(t, x)$ is nondecreasing in $x, g_{x}(t, x)$ is nonincreasing in $x$ for $t \in[0, T], g_{x}\left(t, \alpha_{0}(t)\right) \leq 0$, and for $x \geq y$,

$$
f_{x}(t, x)-f_{x}(t, y) \leq S_{1}\|x-y\|, \quad g_{x}(t, y)-g_{x}(t, x) \leq S_{2}\|x-y\|,
$$

where $S_{1}=\left\{S_{i j}^{(1)}\right\}_{i, j=1}^{n}>0, S_{2}=\left\{S_{i j}^{(2)}\right\}_{i, j=1}^{n}>0$ are constant matrices, $\|\cdot\|$ is a norm in $\mathbb{R}^{n}$.

(3) The functions $I_{k}, G_{k} \in C^{1}\left(D_{k}\left(\alpha_{0}, \beta_{0}\right), \mathbb{R}^{n}\right), I_{k}^{\prime}(x)$ are nondecreasing, $G_{k}^{\prime}(x)$ are nonincreasing, $k=1,2, \ldots, p$, and $I_{k}^{\prime}\left(\alpha_{0}\right) \geq 0, G_{k}^{\prime}\left(\alpha_{0}\right) \leq 0$, and for $x \geq y$,

$$
I_{k}^{\prime}(x)-I_{k}^{\prime}(y) \leq L_{k}\|x-y\|, \quad G_{k}^{\prime}(y)-G_{k}^{\prime}(x) \leq M_{k}\|x-y\|,
$$

where $L_{k}>0, M_{k}>0, k=1,2, \ldots, p$, are constant matrices.

(4) The function $f_{x}\left(t, \alpha_{0}(t)\right) x$ is quasimonotone nondecreasing in $x$ and the function $\left(f_{x}\left(t, \beta_{0}\right)-g_{x}\left(t, \beta_{0}\right)\right) e @ x$ is strictly decreasing in $x$ on $[0, T]$.

(5) The inequalities $\left(I_{k}^{\prime}\left(\beta_{0}\left(\tau_{k}\right)\right)-G_{k}^{\prime}\left(\beta_{0}\left(\tau_{k}\right)\right)\right) e \leq e, k=1,2, \ldots, p$, hold. 
Then there exist two sequences of functions $\left\{\alpha_{m}(t)\right\}_{0}^{\infty}$ and $\left\{\beta_{m}(t)\right\}_{0}^{\infty}$ such that

(a) the sequences are increasing and decreasing correspondingly;

(b) both sequences uniformly converge on the intervals $\left(\tau_{k}, \tau_{k+1}\right]$ to the unique solution of the PBVP (2.1)-(2.2) in $S\left(\alpha_{0}, \beta_{0}\right), k=0,1,2, \ldots, p$;

(c) the convergence is quadratic.

Proof. Consider the periodic boundary value problem for the system of impulsive linear differential equations

$$
\begin{aligned}
& x^{\prime}(t)=f\left(t, \alpha_{0}(t)\right)+g\left(t, \beta_{0}(t)\right)+f_{x}\left(t, \alpha_{0}\right)\left(x-\alpha_{0}\right) \\
& +g_{x}\left(t, \alpha_{0}\right)\left(y-\beta_{0}\right) \quad \text { for } t \in[0, T], t \neq \tau_{k} \text {, } \\
& y^{\prime}(t)=f\left(t, \beta_{0}(t)\right)+g\left(t, \alpha_{0}(t)\right)+f_{x}\left(t, \alpha_{0}\right)\left(y-\beta_{0}\right)+g_{x}\left(t, \alpha_{0}\right)\left(x-\alpha_{0}\right) \text {, } \\
& x\left(\tau_{k}+0\right)=I_{k}\left(\alpha_{0}\left(\tau_{k}\right)\right)+G_{k}\left(\beta_{0}\left(\tau_{k}\right)\right)+I_{k}^{\prime}\left(\alpha_{0}\left(\tau_{k}\right)\right)\left[x\left(\tau_{k}\right)-\alpha_{0}\left(\tau_{k}\right)\right] \\
& +G_{k}^{\prime}\left(\alpha_{0}\left(\tau_{k}\right)\right)\left[y\left(\tau_{k}\right)-\beta_{0}\left(\tau_{k}\right)\right] \text {, } \\
& y\left(\tau_{k}+0\right)=I_{k}\left(\beta_{0}\left(\tau_{k}\right)\right)+G_{k}\left(\alpha_{0}\left(\tau_{k}\right)\right)+I_{k}^{\prime}\left(\alpha_{0}\left(\tau_{k}\right)\right)\left[y\left(\tau_{k}\right)-\beta_{0}\left(\tau_{k}\right)\right] \\
& +G_{k}^{\prime}\left(\alpha_{0}\left(\tau_{k}\right)\right)\left[x\left(\tau_{k}\right)-\alpha_{0}\left(\tau_{k}\right)\right] \text {, } \\
& x(0)=x(T), \quad y(0)=y(T) .
\end{aligned}
$$

The PBVP (3.3) can be written in the form

$$
\begin{gathered}
p^{\prime}=A^{(0)}(t) p(t)+h^{(0)}(t) \quad \text { for } t \in[0, T], t \neq \tau_{k}, \\
p\left(\tau_{k}+0\right)=B_{k}^{(0)} p\left(\tau_{k}\right)+\sigma_{k}^{(0)}, \\
p(0)=p(T),
\end{gathered}
$$

where

$$
\begin{gathered}
p=\left(\begin{array}{l}
x \\
y
\end{array}\right), \quad A^{(0)}(t)=\left(\begin{array}{ll}
f_{x}\left(t, \alpha_{0}\right) & g_{x}\left(t, \alpha_{0}\right) \\
g_{x}\left(t, \alpha_{0}\right) & f_{x}\left(t, \alpha_{0}\right)
\end{array}\right), \\
B_{k}^{(0)}=\left(\begin{array}{cc}
I_{k}^{\prime}\left(\alpha_{0}\left(\tau_{k}\right)\right) & G_{k}^{\prime}\left(\alpha_{0}\left(\tau_{k}\right)\right) \\
G_{k}^{\prime}\left(\alpha_{0}\left(\tau_{k}\right)\right) & I_{k}^{\prime}\left(\alpha_{0}\left(\tau_{k}\right)\right)
\end{array}\right), \\
h^{(0)}(t)=\left(\begin{array}{l}
f\left(t, \alpha_{0}(t)\right)+g\left(t, \beta_{0}(t)\right)-f_{x}\left(t, \alpha_{0}\right) \alpha_{0}-g_{x}\left(t, \alpha_{0}\right) \beta_{0} \\
f\left(t, \beta_{0}(t)\right)+g\left(t, \alpha_{0}(t)\right)-f_{x}\left(t, \alpha_{0}\right) \beta_{0}-g_{x}\left(t, \alpha_{0}\right) \alpha_{0}
\end{array}\right), \\
\sigma_{k}^{(0)}=\left(\begin{array}{l}
I_{k}\left(\alpha_{0}\left(\tau_{k}\right)\right)+G_{k}\left(\beta_{0}\left(\tau_{k}\right)\right)-I_{k}^{\prime}\left(\alpha_{0}\left(\tau_{k}\right)\right) \alpha_{0}\left(\tau_{k}\right)-G_{k}^{\prime}\left(\alpha_{0}\left(\tau_{k}\right)\right) \beta_{0}\left(\tau_{k}\right) \\
I_{k}\left(\beta_{0}\left(\tau_{k}\right)\right)+G_{k}\left(\alpha_{0}\left(\tau_{k}\right)\right)-I_{k}^{\prime}\left(\alpha_{0}\left(\tau_{k}\right)\right) \beta_{0}\left(\tau_{k}\right)-G_{k}^{\prime}\left(\alpha_{0}\left(\tau_{k}\right)\right) \alpha_{0}\left(\tau_{k}\right)
\end{array}\right) .
\end{gathered}
$$

Consider the matrices

$$
C^{0}(t)=\left(\begin{array}{cc}
f_{x}\left(t, \alpha_{0}\right) & -g_{x}\left(t, \alpha_{0}\right) \\
-g_{x}\left(t, \alpha_{0}\right) & f_{x}\left(t, \alpha_{0}\right)
\end{array}\right), \quad D_{k}^{0}=\left(\begin{array}{cc}
I_{k}^{\prime}\left(\alpha_{0}\left(\tau_{k}\right)\right) & -G_{k}^{\prime}\left(\alpha_{0}\left(\tau_{k}\right)\right) \\
-G_{k}^{\prime}\left(\alpha_{0}\left(\tau_{k}\right)\right) & I_{k}^{\prime}\left(\alpha_{0}\left(\tau_{k}\right)\right)
\end{array}\right) .
$$


From conditions (2), (3), (4), and (5) of Theorem 3.1, it follows that $C^{0}(t) \in \Xi$ and $D_{k}^{0} \in \Psi$. According to Lemma $2.8, \operatorname{det}\left(E-C^{0}(t)\right) \neq 0$. Therefore, $\operatorname{det}\left(E-A^{0}(t)\right)=\operatorname{det}(E-$ $\left.C^{0}(t)\right) \neq 0$. Therefore, according to Lemma 2.9, the boundary value problem (3.4) has a unique solution, which can be written in the form (2.21)-(2.22). We denote the solution of (3.4) by $\alpha_{1}(t), \beta_{1}(t)$.

We will prove that $\alpha_{0}(t) \leq \alpha_{1}(t)$ and $\beta_{0}(t) \geq \beta_{1}(t)$ on $[0, T]$. Set $p(t)=\alpha_{0}(t)-\alpha_{1}(t)$, $q(t)=\beta_{1}(t)-\beta_{0}(t)$. Then from the PBVP (3.3) we have

$$
\begin{gathered}
p^{\prime} \leq f_{x}\left(t, \alpha_{0}\right) p-g_{x}\left(t, \alpha_{0}\right) q, \\
q^{\prime} \leq-g_{x}\left(t, \alpha_{0}\right) p+f_{x}\left(t, \alpha_{0}\right) q \quad \text { for } t \in[0, T], t \neq \tau_{k}, \\
p\left(\tau_{k}+0\right) \leq I_{k}^{\prime}\left(\alpha_{0}\left(\tau_{k}\right)\right) p\left(\tau_{k}\right)-G_{k}^{\prime}\left(\alpha_{0}\left(\tau_{k}\right)\right) q\left(\tau_{k}\right), \\
q\left(\tau_{k}+0\right) \leq I_{k}^{\prime}\left(\alpha_{0}\left(\tau_{k}\right)\right) q\left(\tau_{k}\right)-G_{k}^{\prime}\left(\alpha_{0}\left(\tau_{k}\right)\right) p\left(\tau_{k}\right), \\
p(0) \leq p(T), \quad q(0) \leq q(T) .
\end{gathered}
$$

The PBVP (3.9) can be written in the form

$$
\begin{gathered}
m^{\prime}(t) \leq C^{0}(t) m(t) \quad \text { for } t \in[0, T], t \neq \tau_{k}, \\
m\left(\tau_{k}+0\right) \leq D_{k}^{0} m\left(\tau_{k}\right), \\
m(0) \leq m(T)
\end{gathered}
$$

where $m=(p, q)^{T}$ and the matrices $C^{0}(t)$ and $D_{k}^{0}$ are given by (3.8).

From conditions (2), (3), (4), and (5) of Theorem 3.1, it follows that conditions (1) and (2) of Lemma 2.6 are satisfied for $N=2 n$ and therefore $m(t) \leq 0$ on $[0, T]$, that is, $\alpha_{0}(t) \leq \alpha_{1}(t)$ and $\beta_{1}(t) \leq \beta_{0}(t)$ on $[0, T]$.

We will prove that $\alpha_{1}(t) \leq \beta_{1}(t)$. Set $p(t)=\alpha_{1}(t)-\beta_{1}(t)$. Then, from the PBVP (3.3), conditions (2) and (3), and Lemma 2.11, we have

$$
\begin{gathered}
p^{\prime} \leq\left[f_{x}\left(t, \alpha_{0}\right)-g_{x}\left(t, \alpha_{0}\right)\right] p \quad \text { for } t \in[0, T], t \neq \tau_{k}, \\
p\left(\tau_{k}+0\right) \leq\left[I_{k}^{\prime}\left(\alpha_{0}\left(\tau_{k}\right)\right)-G_{k}^{\prime}\left(\alpha_{0}\left(\tau_{k}\right)\right)\right] p\left(\tau_{k}\right), \\
p(0) \leq p(T) .
\end{gathered}
$$

According to Lemma 2.6, for $N=n, A(t)=f_{x}\left(t, \alpha_{0}\right)-g_{x}\left(t, \alpha_{0}\right), B_{k}=I_{k}^{\prime}\left(\alpha_{0}\left(\tau_{k}\right)\right)-G_{k}^{\prime}\left(\alpha_{0}\left(\tau_{k}\right)\right)$, we have $p(t) \leq 0$ on $[0, T]$.

Assume that for some $m$ the functions $\alpha_{m}(t)$ and $\beta_{m}(t)$ are constructed such that $\alpha_{m-1}(t) \leq \alpha_{m}(t) \leq \beta_{m}(t) \leq \beta_{m-1}(t)$. Consider the boundary value problem for the system of linear impulsive differential equations

$$
\begin{aligned}
x^{\prime}(t)= & f\left(t, \alpha_{m}(t)\right)+g\left(t, \beta_{m}(t)\right)+f_{x}\left(t, \alpha_{m}\right)\left(x-\alpha_{m}\right) \\
& +g_{x}\left(t, \alpha_{m}\right)\left(y-\beta_{m}\right) \quad \text { for } t \in[0, T], t \neq \tau_{k}, \\
y^{\prime}(t)= & f\left(t, \beta_{m}(t)\right)+g\left(t, \alpha_{m}(t)\right)+f_{x}\left(t, \alpha_{m}\right)\left(y-\beta_{m}\right)+g_{x}\left(t, \alpha_{m}\right)\left(x-\alpha_{m}\right),
\end{aligned}
$$




$$
\begin{aligned}
& x\left(\tau_{k}+0\right)= I_{k}\left(\alpha_{m}\left(\tau_{k}\right)\right)+G_{k}\left(\beta_{m}\left(\tau_{k}\right)\right)+I_{k}^{\prime}\left(\alpha_{m}\left(\tau_{k}\right)\right)\left[x\left(\tau_{k}\right)-\alpha_{m}\left(\tau_{k}\right)\right] \\
&+G_{k}^{\prime}\left(\alpha_{m}\left(\tau_{k}\right)\right)\left[y\left(\tau_{k}\right)-\beta_{m}\left(\tau_{k}\right)\right], \\
& y\left(\tau_{k}+0\right)= I_{k}\left(\beta_{m}\left(\tau_{k}\right)\right)+G_{k}\left(\alpha_{m}\left(\tau_{k}\right)\right)+I_{k}^{\prime}\left(\alpha_{m}\left(\tau_{k}\right)\right)\left[y\left(\tau_{k}\right)-\beta_{m}\left(\tau_{k}\right)\right] \\
&+G_{k}^{\prime}\left(\alpha_{m}\left(\tau_{k}\right)\right)\left[x\left(\tau_{k}\right)-\alpha_{m}\left(\tau_{k}\right)\right], \\
& x(0)=x(T), \quad y(0)=y(T),
\end{aligned}
$$

which can be written in the form

$$
\begin{gathered}
p_{m+1}^{\prime}=A^{(m)}(t) p_{m+1}(t)+h^{(m)}(t) \quad \text { for } t \in[0, T], t \neq \tau_{k} \\
p_{m+1}\left(\tau_{k}+0\right)=B_{k}^{(m)} p_{m+1}\left(\tau_{k}\right)+\sigma_{k}^{(m)} \\
p_{m+1}(0)=p_{m+1}(T)
\end{gathered}
$$

where

$$
\begin{gathered}
p_{m+1}=\left(\begin{array}{c}
\alpha_{m+1} \\
\beta_{m+1}
\end{array}\right), \quad A^{(m)}(t)=\left(\begin{array}{ll}
f_{x}\left(t, \alpha_{m}\right) & g_{x}\left(t, \alpha_{m}\right) \\
g_{x}\left(t, \alpha_{m}\right) & f_{x}\left(t, \alpha_{m}\right)
\end{array}\right), \\
B_{k}^{(m)}=\left(\begin{array}{cc}
I_{k}^{\prime}\left(\alpha_{m}\left(\tau_{k}\right)\right) & G_{k}^{\prime}\left(\alpha_{m}\left(\tau_{k}\right)\right) \\
G_{k}^{\prime}\left(\alpha_{m}\left(\tau_{k}\right)\right) & I_{k}^{\prime}\left(\alpha_{m}\left(\tau_{k}\right)\right)
\end{array}\right), \\
h^{(m)}(t)=\left(\begin{array}{l}
f\left(t, \alpha_{m}(t)\right)+g\left(t, \beta_{m}(t)\right)-f_{x}\left(t, \alpha_{m}\right) \alpha_{m}-g_{x}\left(t, \alpha_{m}\right) \beta_{m} \\
f\left(t, \beta_{m}(t)\right)+g\left(t, \alpha_{m}(t)\right)-f_{x}\left(t, \alpha_{m}\right) \beta_{m}-g_{x}\left(t, \alpha_{m}\right) \alpha_{m}
\end{array}\right), \\
\sigma_{k}^{(m)}=\left(\begin{array}{l}
I_{k}\left(\alpha_{m}\left(\tau_{k}\right)\right)+G_{k}\left(\beta_{m}\left(\tau_{k}\right)\right)-I_{k}^{\prime}\left(\alpha_{m}\left(\tau_{k}\right)\right) \alpha_{m}\left(\tau_{k}\right)-G_{k}^{\prime}\left(\alpha_{m}\left(\tau_{k}\right)\right) \beta_{m}\left(\tau_{k}\right) \\
I_{k}\left(\beta_{m}\left(\tau_{k}\right)\right)+G_{k}\left(\alpha_{m}\left(\tau_{k}\right)\right)-I_{k}^{\prime}\left(\alpha_{m}\left(\tau_{k}\right)\right) \beta_{m}\left(\tau_{k}\right)-G_{k}^{\prime}\left(\alpha_{m}\left(\tau_{k}\right)\right) \alpha_{m}\left(\tau_{k}\right)
\end{array}\right) .
\end{gathered}
$$

Consider the matrices

$$
C^{(m)}(t)=\left(\begin{array}{cc}
f_{x}\left(t, \alpha_{m}\right) & -g_{x}\left(t, \alpha_{m}\right) \\
-g_{x}\left(t, \alpha_{m}\right) & f_{x}\left(t, \alpha_{m}\right)
\end{array}\right), \quad D_{k}^{(m)}=\left(\begin{array}{cc}
I_{k}^{\prime}\left(\alpha_{m}\left(\tau_{k}\right)\right) & -G_{k}^{\prime}\left(\alpha_{m}\left(\tau_{k}\right)\right) \\
-G_{k}^{\prime}\left(\alpha_{m}\left(\tau_{k}\right)\right) & I_{k}^{\prime}\left(\alpha_{m}\left(\tau_{k}\right)\right)
\end{array}\right)
$$

From the inequality $\alpha_{m-1}(t) \leq \alpha_{m}(t)$, the monotonicity of the derivatives $f_{x}, g_{x}$, and conditions (4) and (5) of Theorem 3.1, it follows that $C^{(m)}(t) \in \Xi$ and $D_{k}^{(m)} \in \Psi, k=$ $1,2, \ldots, p$. Therefore, according to Lemma $2.8, \operatorname{det}\left(E-C^{(m)}(t)\right) \neq 0$ and therefore $\operatorname{det}(E-$ $\left.A^{(m)}(t)\right)=\operatorname{det}\left(E-C^{(m)}(t)\right) \neq 0$. Applying Lemma 2.9, we obtain that the PBVP (3.13)(3.15) has a unique solution $\alpha_{m+1}(t), \beta_{m+1}(t)$.

We can prove in the same way as for the function $\alpha_{1}(t)$ and $\beta_{1}(t)$ that $\alpha_{m}(t) \leq \alpha_{m+1}(t) \leq$ $\beta_{m+1}(t) \leq \beta_{m}(t)$.

We will prove the convergence of the sequences $\left\{\alpha_{m}(t)\right\}_{0}^{\infty}$ and $\left\{\beta_{m}(t)\right\}_{0}^{\infty}$. 
Consider the interval $\left[0, \tau_{1}\right]$. From $(3.13)-(3.15)$, the functions $\alpha_{m}, \beta_{m}$ satisfy on $\left[0, \tau_{1}\right]$ the integral equation

$$
p_{m+1}(t)=p_{m+1}(0)+\int_{0}^{t}\left(A^{(m)}(s) p_{m+1}(s)+h^{(m)}(s)\right) d s .
$$

The sequences $\left\{\alpha_{m}(t)\right\}$ and $\left\{\beta_{m}(t)\right\}$ are uniformly bounded and equicontinuous on the interval $\left[0, \tau_{1}\right]$ and therefore they are uniformly convergent on this interval.

Denote

$$
\lim _{m \rightarrow \infty} \alpha_{m}(t)=u^{(1)}(t), \quad \lim _{m \rightarrow \infty} \beta_{m}(t)=v^{(1)}(t), \quad t \in\left[0, \tau_{1}\right]
$$

From the uniform convergence and the definition of the functions $\alpha_{m}(t)$ and $\beta_{m}(t)$, the validity of the inequalities $\alpha_{0}(t) \leq u^{(1)}(t) \leq v^{(1)}(t) \leq \beta_{0}(t), t \in\left[0, \tau_{1}\right]$, follows.

Taking a limit into the integral equation (3.18) for $t \in\left[0, \tau_{1}\right]$, we obtain

$$
w^{(1)}(t)=w^{(1)}(0)+\int_{0}^{t}\left(A_{1}(s) w^{(1)}(s)+h_{1}(s)\right) d s,
$$

where

$$
\begin{gathered}
w^{(1)}=\left(\begin{array}{l}
u^{(1)} \\
v^{(1)}
\end{array}\right), \quad A_{1}(t)=\lim _{m \rightarrow \infty} A^{(m)}(t)=\left(\begin{array}{ll}
f_{x}\left(t, u^{(1)}\right) & g_{x}\left(t, u^{(1)}\right) \\
g_{x}\left(t, u^{(1)}\right) & f_{x}\left(t, u^{(1)}\right)
\end{array}\right), \\
h_{1}(t)=\lim _{m \rightarrow \infty} h^{(m)}(t)=\left(\begin{array}{l}
f\left(t, u^{(1)}(t)\right)+g\left(t, v^{(1)}(t)\right)-f_{x}\left(t, u^{(1)}\right) u^{(1)}-g_{x}\left(t, u^{(1)}\right) v^{(1)} \\
f\left(t, v^{(1)}(t)\right)+g\left(t, u^{(1)}(t)\right)-f_{x}\left(t, u^{(1)}\right) v^{(1)}-g_{x}\left(t, u^{(1)}\right) u^{(1)}
\end{array}\right) .
\end{gathered}
$$

From the definition of the matrix $A_{1}(t)$ and the function $h_{1}(t)$, we have

$$
A_{1}(t) w^{(1)}(t)+h_{1}(t)=\left(\begin{array}{l}
f\left(t, u^{(1)}(t)\right)+g\left(t, v^{(1)}(t)\right) \\
f\left(t, v^{(1)}(t)\right)+g\left(t, u^{(1)}(t)\right)
\end{array}\right) .
$$

From (3.22) it follows that $\left(w^{(1)}\right)^{\prime}=A_{1}(t) w^{(1)}+h_{1}(t)$ on $\left[0, \tau_{1}\right]$.

Consider the interval $\left[\tau_{1}+0, \tau_{2}\right]$. From (3.13), it follows that the functions $\alpha_{m}, \beta_{m}$ satisfy on $\left[\tau_{1}+0, \tau_{2}\right]$ the integral equation

$$
p_{m+1}(t)=p_{m+1}\left(\tau_{1}+0\right)+\int_{\tau_{1}}^{t}\left(A^{(m)}(s) p_{m+1}(s)+h^{(m)}(s)\right) d s .
$$

On this interval, the sequences $\left\{\alpha_{m}(t)\right\}_{0}^{\infty}$ and $\left\{\beta_{m}(t)\right\}_{0}^{\infty}$ are uniformly bounded and equicontinuous and therefore they are uniformly convergent. Denote

$$
\lim _{m \rightarrow \infty} \alpha_{m}(t)=u^{(2)}(t), \quad \lim _{m \rightarrow \infty} \beta_{m}(t)=v^{(2)}(t), \quad t \in\left[\tau_{1}+0, \tau_{2}\right]
$$

From the uniform convergence and the definition of the functions $\alpha_{m}(t)$ and $\beta_{m}(t)$, the validity of the inequalities $\alpha_{0}(t) \leq u^{(2)}(t) \leq v^{(2)}(t) \leq \beta_{0}(t)$ on $\left[\tau_{1}+0, \tau_{2}\right]$ follows. 
Taking limits into (3.14) and (3.23), we obtain that $w^{(2)}\left(\tau_{1}\right)=B_{1} w^{(1)}\left(\tau_{1}\right)$ and

$$
\begin{aligned}
w^{(2)}(t) & =w^{(2)}\left(\tau_{1}+0\right)+\int_{\tau_{1}}^{t}\left(A_{2}(s) w^{(2)}(s)+h_{2}(s)\right) d s \\
& =B_{1} w^{(1)}\left(\tau_{1}\right)+\sigma_{1}+\int_{\tau_{1}}^{t}\left(A_{2}(s) w^{(2)}(s)+h_{2}(s)\right) d s
\end{aligned}
$$

where

$$
\begin{gathered}
w^{(2)}=\left(\begin{array}{l}
u^{(2)} \\
v^{(2)}
\end{array}\right), \quad A_{2}(t)=\lim _{m \rightarrow \infty} A^{(m)}(t)=\left(\begin{array}{ll}
f_{x}\left(t, u^{(2)}\right) & g_{x}\left(t, u^{(2)}\right) \\
g_{x}\left(t, u^{(2)}\right) & f_{x}\left(t, u^{(2)}\right)
\end{array}\right), \\
B_{1}=\lim _{m \rightarrow \infty} B_{1}^{(m)}=\left(\begin{array}{cc}
I_{1}^{\prime}\left(u^{(1)}\left(\tau_{1}\right)\right) & G_{1}^{\prime}\left(u^{(1)}\left(\tau_{1}\right)\right) \\
G_{1}^{\prime}\left(u^{(1)}\left(\tau_{1}\right)\right) & I_{1}^{\prime}\left(u^{(1)}\left(\tau_{1}\right)\right)
\end{array}\right), \\
\sigma_{1}=\left(\begin{array}{c}
I_{1}\left(u^{(1)}\left(\tau_{1}\right)\right)+G_{1}\left(v^{(1)}\left(\tau_{1}\right)\right)-I_{1}^{\prime}\left(u^{(1)}\left(\tau_{1}\right)\right) u^{(1)}\left(\tau_{1}\right)-G_{1}^{\prime}\left(u^{(1)}\left(\tau_{1}\right)\right) v^{(1)}\left(\tau_{1}\right) \\
I_{1}\left(v^{(1)}\left(\tau_{1}\right)\right)+G_{1}\left(u^{(1)}\left(\tau_{1}\right)\right)-I_{1}^{\prime}\left(u^{(1)}\left(\tau_{1}\right)\right) v^{(1)}\left(\tau_{1}\right)-G_{1}^{\prime}\left(u^{(1)}\left(\tau_{1}\right)\right) u^{(1)}\left(\tau_{1}\right)
\end{array}\right), \\
h_{1}(t)=\left(\begin{array}{l}
f\left(t, u^{(2)}(t)\right)+g\left(t, v^{(2)}(t)\right)-f_{x}\left(t, u^{(2)}\right) u^{(2)}-g_{x}\left(t, u^{(2)}\right) v^{(2)} \\
f\left(t, v^{(2)}(t)\right)+g\left(t, u^{(2)}(t)\right)-f_{x}\left(t, u^{(2)}\right) v^{(2)}-g_{x}\left(t, u^{(2)}\right) u^{(2)}
\end{array}\right) .
\end{gathered}
$$

By induction, we prove that on each interval $\left[\tau_{k}+0, \tau_{k+1}\right]$ the sequences $\left\{\alpha_{m}(t)\right\}_{0}^{\infty}$ and $\left\{\beta_{m}(t)\right\}_{0}^{\infty}$ are uniformly convergent, where $k=0,1,2, \ldots, p$. Their limits $u^{(k+1)}(t)$ and $v^{(k+1)}(t)$ satisfy the relations $u^{(k+1)}, v^{(k+1)} \in S\left(\alpha_{0}, \beta_{0}\right), u^{(k+1)} \leq v^{(k+1)}$ on $\left[\tau_{k}, \tau_{k+1}\right]$, $w^{(k+1)}\left(\tau_{k}\right)=B_{k} w^{(k)}\left(\tau_{k}\right)+\sigma_{k}$, and they are the solution of the linear integral equations

$$
\begin{aligned}
w^{(k+1)}(t) & =w^{(k+1)}\left(\tau_{k}\right)+\int_{\tau_{k}}^{t}\left(A_{k+1}(s) w^{(k+1)}(s)+h_{k+1}(s)\right) d s \\
& =B_{k} w^{(k)}\left(\tau_{k}\right)+\sigma_{k}+\int_{\tau_{k}}^{t}\left(A_{k+1}(s) w^{(k+1)}(s)+h_{k+1}(s)\right) d s
\end{aligned}
$$

where

$$
\begin{gathered}
w^{(k+1)}=\left(\begin{array}{c}
u^{(k+1)} \\
v^{(k+1)}
\end{array}\right), \quad A_{k+1}(t)=\lim _{m \rightarrow \infty} A^{(m)}(t)=\left(\begin{array}{ll}
f_{x}\left(t, u^{(k+1)}\right) & g_{x}\left(t, u^{(k+1)}\right) \\
g_{x}\left(t, u^{(k+1)}\right) & f_{x}\left(t, u^{(k+1)}\right)
\end{array}\right), \\
B_{k}=\lim _{m \rightarrow \infty} B_{k}^{(m)}=\left(\begin{array}{cc}
I_{k}^{\prime}\left(u^{(k)}\left(\tau_{k}\right)\right) & G_{k}^{\prime}\left(u^{(k)}\left(\tau_{k}\right)\right) \\
G_{k}^{\prime}\left(u^{(k)}\left(\tau_{k}\right)\right) & I_{k}^{\prime}\left(u^{(k)}\left(\tau_{k}\right)\right)
\end{array}\right), \\
\sigma_{k}=\left(\begin{array}{l}
I_{k}\left(u^{(k)}\left(\tau_{k}\right)\right)+G_{k}\left(v^{(k)}\left(\tau_{k}\right)\right)-I_{k}^{\prime}\left(u^{(k)}\left(\tau_{k}\right)\right) u^{(k)}\left(\tau_{k}\right)-G_{k}^{\prime}\left(u^{(k)}\left(\tau_{k}\right)\right) v^{(k)}\left(\tau_{k}\right) \\
I_{k}\left(v^{(k)}\left(\tau_{k}\right)\right)+G_{k}\left(u^{(k)}\left(\tau_{k}\right)\right)-I_{k}^{\prime}\left(u^{(k)}\left(\tau_{k}\right)\right) v^{(k)}\left(\tau_{k}\right)-G_{k}^{\prime}\left(u^{(k)}\left(\tau_{k}\right)\right) u^{(k)}\left(\tau_{k}\right)
\end{array}\right), \\
h_{k}(t)=\left(\begin{array}{l}
f\left(t, u^{(k+1)}(t)\right)+g\left(t, v^{(k+1)}(t)\right)-f_{x}\left(t, u^{(k+1)}\right) u^{(k+1)}-g_{x}\left(t, u^{(k+1)}\right) v^{(k+1)} \\
f\left(t, v^{(k+1)}(t)\right)+g\left(t, u^{(k+1)}(t)\right)-f_{x}\left(t, u^{(k+1)}\right) v^{(k+1)}-g_{x}\left(t, u^{(k+1)}\right) u^{(k+1)}
\end{array}\right) .
\end{gathered}
$$


From the equalities (3.28), we obtain that

$$
\begin{gathered}
A_{k+1}(t) w^{(k+1)}(t)+h_{k+1}(t)=\left(\begin{array}{l}
f\left(t, u^{(k+1)}(t)\right)+g\left(t, v^{(k+1)}(t)\right) \\
f\left(t, v^{(k+1)}(t)\right)+g\left(t, u^{(k+1)}(t)\right)
\end{array}\right), \\
B_{k} w^{(k)}\left(\tau_{k}\right)+\sigma_{k}=\left(\begin{array}{l}
I_{k}\left(u^{(k)}\left(\tau_{k}\right)\right)+G_{k}\left(v^{(k)}\left(\tau_{k}\right)\right) \\
G_{k}\left(u^{(k)}\left(\tau_{k}\right)\right)+I_{k}\left(v^{(k)}\left(\tau_{k}\right)\right)
\end{array}\right) .
\end{gathered}
$$

Define the piecewise continuous functions $u, v \in P C\left([0, T], R^{n}\right)$ by the equalities $u(t)=$ $u^{(k+1)}(t)$ and $v(t)=v^{(k+1)}(t)$ for $t \in\left(\tau_{k}, \tau_{k+1}\right], k=0,1,2, \ldots, p$. From the properties of the functions $u^{(k+1)}(t)$ and $v^{(k+1)}(t)$, it follows that $u, v \in S\left(\alpha_{0}, \beta_{0}\right)$ and $u(t) \leq v(t)$ on $[0, T]$. By considering the function $w=(u, v)$, it follows

$$
w\left(\tau_{k}+0\right)=\lim _{t \downarrow \tau_{k}} w^{(k+1)}(t)=w^{(k+1)}\left(\tau_{k}\right)=B_{k} w^{k}\left(\tau_{k}\right)+\sigma_{k}=B_{k} w\left(\tau_{k}\right)+\sigma_{k}
$$

From the integral equations (3.27), it follows that the function $w(t)$ satisfies the integral equation

$$
w(t)=w\left(\tau_{k}+0\right)+\int_{\tau_{k}}^{t}(A(s) w(s)+h(s)) d s, \quad t \in\left[\tau_{k}+0, \tau_{k+1}\right]
$$

Taking the limit into the equality (3.15), we obtain that $w^{(1)}(0)=w^{(p+1)}(T)$ or

$$
w(0)=w(T)
$$

From the equalities (3.29), we obtain that for $k=0,1,2, \ldots, p$,

$$
\begin{aligned}
A_{k+1}(t) w^{(k+1)}(t)+h_{k+1}(t) & =\left(\begin{array}{l}
f(t, u(t))+g(t, v(t)) \\
f(t, v(t))+g(t, u(t))
\end{array}\right), \\
B_{k} w^{(k)}\left(\tau_{k}\right)+\sigma_{k} & =\left(\begin{array}{l}
I_{k}\left(u\left(\tau_{k}\right)\right)+G_{k}\left(v\left(\tau_{k}\right)\right) \\
G_{k}\left(u\left(\tau_{k}\right)\right)+I_{k}\left(v\left(\tau_{k}\right)\right)
\end{array}\right) .
\end{aligned}
$$

From (3.30), (3.31), (3.32), and (3.33), it follows that the functions $u(t)$ and $v(t)$ are solutions of the PBVP

$$
\begin{gathered}
u^{\prime}=f(t, u(t))+g(t, v(t)), \quad v^{\prime}=f(t, v(t))+g(t, u(t)) \quad \text { for } t \in[0, T], t \neq \tau_{k}, \\
u\left(\tau_{k}+0\right)=I_{k}\left(u\left(\tau_{k}\right)\right)+G_{k}\left(v\left(\tau_{k}\right)\right), \quad v\left(\tau_{k}+0\right)=I_{k}\left(v\left(\tau_{k}\right)\right)+G_{k}\left(u\left(\tau_{k}\right)\right), \\
u(0)=u(T), \quad v(0)=v(T) .
\end{gathered}
$$


Consider the function $p(t)=v(t)-u(t) \geq 0$. From the PBVP (3.34) and the properties of the derivatives of the functions $f, g$, we obtain that

$$
\begin{gathered}
p^{\prime}=\int_{0}^{1} f_{x}(s, \lambda v+(1-\lambda) u) p-\int_{0}^{1} g_{x}(s, \lambda v+(1-\lambda) u) p \leq\left(f_{x}\left(t, \beta_{0}\right)-g_{x}\left(t, \beta_{0}\right)\right) p \\
p\left(\tau_{k}+0\right)=\left(I_{k}^{\prime}\left(\beta_{0}\left(\tau_{k}\right)\right)-G_{k}^{\prime}\left(\beta_{0}\left(\tau_{k}\right)\right)\right) p\left(\tau_{k}\right), \quad p(0)=p(T) .
\end{gathered}
$$

According to Lemma 2.6, for $N=n, A(t)=f_{x}\left(t, \beta_{0}\right)-g_{x}\left(t, \beta_{0}\right), B_{k}=I_{k}^{\prime}\left(\beta_{0}\left(\tau_{k}\right)\right)-G_{k}^{\prime}\left(\beta_{0}\left(\tau_{k}\right)\right)$, we have $p(t) \leq 0$ on $[0, T]$. Therefore, $p(t)=0$, which proves that $u(t)=v(t)$.

We will prove that the convergence is quadratic.

Define the functions $p_{m+1}(t)=u(t)-\alpha_{m+1}(t)$ and $q_{m+1}(t)=\beta_{m+1}(t)-u(t), t \in[0, T]$. For $t \in[0, T], t \neq \tau_{k}$, we obtain the inequalities

$$
\begin{aligned}
p_{m+1}^{\prime} \leq & f(t, u)-f\left(t, \alpha_{m}\right)+g(t, u)-g\left(t, \beta_{m}\right) \\
& -f_{x}\left(t, \alpha_{m}\right)\left(p_{m}-p_{m+1}\right)-g_{x}\left(t, \alpha_{m}\right)\left(q_{m+1}-q_{m}\right) \\
= & \left(\int_{0}^{1} f_{x}\left(t, \lambda u+(1-\lambda) \alpha_{m}\right) d \lambda\right) p_{m}-\left(\int_{0}^{1} g_{x}\left(t, \lambda u+(1-\lambda) \beta_{m}\right) d \lambda\right) q_{m} \\
& -f_{x}\left(t, \alpha_{m}\right)\left(p_{m}-p_{m+1}\right)-g_{x}\left(t, \alpha_{m}\right)\left(q_{m+1}-q_{m}\right) \\
\leq & \left(f_{x}(t, u)-f_{x}\left(t, \alpha_{m}\right)\right) p_{m}+\left(g_{x}\left(t, \alpha_{m}\right)-g_{x}\left(t, \beta_{m}\right)\right) q_{m} \\
& +f_{x}\left(t, \alpha_{m}\right) p_{m+1}-g_{x}\left(t, \alpha_{m}\right) q_{m+1} \\
\leq & f_{x}\left(t, \alpha_{m}\right) p_{m+1}-g_{x}\left(t, \alpha_{m}\right) q_{m+1}+S_{1}\left\|p_{m}\right\| p_{m}+S_{2}\left\|\alpha_{m}-\beta_{m}\right\| q_{m} \\
\leq & f_{x}\left(t, \alpha_{m}\right) p_{m+1}-g_{x}\left(t, \alpha_{m}\right) q_{m+1}+\left(\tilde{s}+\frac{1}{2} \tilde{m}\right)\left\|p_{m}\right\|^{2}+\frac{3}{2} \tilde{m}\left\|q_{m}\right\|^{2},
\end{aligned}
$$

where $\tilde{s}=\left(\widetilde{s_{1}}, \widetilde{s_{2}}, \ldots, \widetilde{s_{n}}\right), \tilde{m}=\left(\widetilde{m_{1}}, \widetilde{m_{2}}, \ldots, \widetilde{m_{n}}\right), \widetilde{s_{i}}=\sum_{j=1}^{n} S_{i j}^{(1)}, \widetilde{m_{i}}=\sum_{j=1}^{n} S_{i j}^{(2)}, i=1,2, \ldots, n$.

For $t \in[0, T], t \neq \tau_{k}$, from the definition of the functions $\alpha_{m}(t)$ and $\beta_{m}(t)$ and the fact that $u(t)$ is a solution of the PBVP (2.1)-(2.2), we obtain

$$
\begin{aligned}
q_{m+1}^{\prime} \leq & f\left(t, \beta_{m}\right)-f(t, u)+g\left(t, \alpha_{m}\right)-g(t, u) \\
& -f_{x}\left(t, \alpha_{m}\right)\left(q_{m}-q_{m+1}\right)-g_{x}\left(t, \alpha_{m}\right)\left(p_{m+1}-p_{m}\right) \\
= & \left(\int_{0}^{1} f_{x}\left(t, \lambda \beta_{m}+(1-\lambda) u\right) d \lambda\right) q_{m}-\left(\int_{0}^{1} g_{x}\left(t, \lambda \alpha_{m}+(1-\lambda) u\right) d \lambda\right) p_{m} \\
& -f_{x}\left(t, \alpha_{m}\right)\left(q_{m}-q_{m+1}\right)-g_{x}\left(t, \alpha_{m}\right)\left(p_{m+1}-p_{m}\right) \\
\leq & \left(f_{x}\left(t, \beta_{m}\right)-f_{x}\left(t, \alpha_{m}\right)\right) q_{m}+\left(g_{x}\left(t, \alpha_{m}\right)-g_{x}(t, u)\right) p_{m} \\
& +f_{x}\left(t, \alpha_{m}\right) q_{m+1}-g_{x}\left(t, \alpha_{m}\right) p_{m+1} \\
\leq & -g_{x}\left(t, \alpha_{m}\right) p_{m+1}+f_{x}\left(t, \alpha_{m}\right) q_{m+1}+S_{1}\left\|p_{m}+q_{m}\right\| q_{m}+S_{2}\left\|p_{m}\right\| p_{m} \\
\leq & -g_{x}\left(t, \alpha_{m}\right) p_{m+1}+f_{x}\left(t, \alpha_{m}\right) q_{m+1}+\frac{3}{2} \tilde{s}\left\|q_{m}\right\|^{2}+\left(\tilde{m}+\frac{1}{2} \tilde{s}\right)\left\|p_{m}\right\|^{2} .
\end{aligned}
$$


14 Method impulsive systems with PB conditions

For $k=1,2, \ldots, p$, from the impulsive conditions, we have

$$
\begin{aligned}
& p_{m+1}\left(\tau_{k}+0\right) \\
& \leq I_{k}\left(u\left(\tau_{k}\right)\right)-I_{k}\left(\alpha_{m}\left(\tau_{k}\right)\right)+G_{k}\left(u\left(\tau_{k}\right)\right)-G_{k}\left(\alpha_{m}\left(\tau_{k}\right)\right) \\
& \quad+I_{k}^{\prime}\left(\alpha_{m}\left(\tau_{k}\right)\right)\left[\alpha_{m+1}\left(\tau_{k}\right)-\alpha_{m}\left(\tau_{k}\right)\right]-G_{k}\left(\alpha_{m}\left(\tau_{k}\right)\right)\left[\beta_{m+1}\left(\tau_{k}\right)-\beta_{m}\left(\tau_{k}\right)\right] \\
& \leq I_{k}^{\prime}\left(\alpha_{m}\left(\tau_{k}\right)\right) p_{m+1}\left(\tau_{k}\right)-G_{k}\left(\alpha_{m}\left(\tau_{k}\right)\right) q_{m+1}\left(\tau_{k}\right) \\
& \quad+\mu_{1}\left\|p_{m}\left(\tau_{k}\right)\right\|^{2}+\mu_{2}\left\|q_{m}\left(\tau_{k}\right)\right\|^{2}, \\
& q_{m+1}\left(\tau_{k}+0\right) \leq-G_{k}\left(\alpha_{m}\left(\tau_{k}\right)\right) p_{m+1}\left(\tau_{k}\right)+I_{k}^{\prime}\left(\alpha_{m}\left(\tau_{k}\right)\right) q_{m+1}\left(\tau_{k}\right) \\
& \quad+\widetilde{\mu_{1}}\left\|p_{m}\left(\tau_{k}\right)\right\|^{2}+\widetilde{\mu_{2}}\left\|q_{m}\left(\tau_{k}\right)\right\|^{2},
\end{aligned}
$$

where $\mu_{i}, \tilde{\mu}_{i}, i=1,2$ are constant vectors.

The differential inequalities (3.36)-(3.39) can be written in the form

$$
\begin{gathered}
r_{m+1}^{\prime} \leq A(t) r_{m+1}+P\left\|r_{m}\right\|^{2}, \quad t \neq \tau_{k}, \\
r_{m+1}\left(\tau_{k}+0\right) \leq B_{k} r_{m+1}\left(\tau_{k}\right)+Q_{k}\left\|r_{m}\left(\tau_{k}\right)\right\|^{2}, \quad r_{m+1}(0)=r_{m+1}(T),
\end{gathered}
$$

where

$$
\begin{gathered}
r_{m+1}=\left(\begin{array}{c}
p_{m+1} \\
q_{m+1}
\end{array}\right), \quad P=\left(\begin{array}{c}
\tilde{s}+2 \tilde{m} \\
2 \tilde{s}+\tilde{m}
\end{array}\right), \quad Q_{k}=\left(\begin{array}{c}
\mu_{1}+\mu_{2} \\
\widetilde{\mu_{1}}+\widetilde{\mu_{2}}
\end{array}\right), \\
A(t)=\left(\begin{array}{cc}
f_{x}\left(t, \alpha_{m}\right) & -g_{x}\left(t, \alpha_{m}\right) \\
-g_{x}\left(t, \alpha_{m}\right) & f_{x}\left(t, \alpha_{m}\right)
\end{array}\right), \quad B_{k}=\left(\begin{array}{cc}
I_{k}^{\prime}\left(\alpha_{m}\left(\tau_{k}\right)\right) & -G_{k}^{\prime}\left(\alpha_{m}\left(\tau_{k}\right)\right) \\
-G_{k}^{\prime}\left(\alpha_{m}\left(\tau_{k}\right)\right) & I_{k}^{\prime}\left(\alpha_{m}\left(\tau_{k}\right)\right)
\end{array}\right) .
\end{gathered}
$$

From the monotonicity of the functions $f_{x}(t, x)$ and $g_{x}(t, x)$, the inequalities $\alpha_{m}(t) \geq$ $\alpha_{0}(t), \beta_{m}(t) \leq \beta_{0}(t)$, and condition (4) of Theorem 3.1, it follows that condition (1) of Lemma 2.8 is satisfied. The matrices $B_{k}$ according to conditions (3) and (5) of Theorem 3.1 are from the class $\Psi$. According to Lemma 2.8, the inequality

$$
r_{m+1}(t) \leq W(t, 0) x_{0}+\int_{0}^{t} W(t, s) P\left\|r_{m}(s)\right\|^{2} d s+\sum_{0<t_{k}<t} W\left(t, t_{k}+0\right) Q_{k}\left\|r_{m}\left(\tau_{k}\right)\right\|^{2}
$$

holds for $t \in[0, T]$, where

$$
x_{0}=(E-W(T, 0))^{-1}\left(\int_{0}^{T} W(T, s) P\left\|r_{m}(s)\right\|^{2} d s+\sum_{k=1}^{p} W\left(T, t_{k}+0\right) Q_{k}\left\|r_{m}\left(\tau_{k}\right)\right\|^{2}\right)
$$

and $W(t, s)$ is given by $(2.17)$.

From the inequalities (3.42), (3.43), it follows that there exists a number $\lambda>0$ such that $\left\|\left|r_{m+1}\right|\right\||\leq \lambda| \| r_{m}||^{2}$, where $\left|\|r \mid\|=\sup _{t \in[0, T]}\|r(t)\|\right.$. This inequality proves the quadratic convergence. 
The next theorem is for the case when the lower and upper solutions are completely opposite to those in Theorem 3.1.

Theorem 3.2. Let the following conditions hold.

(1) The functions $\alpha_{0}(t), \beta_{0}(t) \in P C^{1}\left([0, T], \mathbb{R}^{n}\right), \alpha_{0}(t) \leq \beta_{0}(t)$ for $t \in[0, T]$, are coupled lower and upper solutions of the PBVP (2.1)-(2.2) of type II.

(2) The functions $f_{x}, g_{x}$ exist and are continuous on $\Omega\left(\alpha_{0}, \beta_{0}\right), f_{x}(t, x)$ is nondecreasing in $x, g_{x}(t, x)$ is nonincreasing in $x$ for $t \in[0, T], f_{x}\left(t, \beta_{0}(t)\right) \leq 0$, and for $x \geq y$,

$$
f_{x}(t, x)-f_{x}(t, y) \leq S_{1}\|x-y\|, \quad g_{x}(t, y)-g_{x}(t, x) \leq S_{2}\|x-y\|,
$$

where $S_{1}>0, S_{2}>0$ are constant matrices, $\|\cdot\|$ is a norm in $\mathbb{R}^{n}$.

(3) The functions $I_{k}, G_{k} \in C^{1}\left(D_{k}\left(\alpha_{0}, \beta_{0}\right), \mathbb{R}^{n}\right), I_{k}^{\prime}(x)$ are nondecreasing, $G_{k}^{\prime}(x)$ are nonincreasing, $k=1,2, \ldots, p$ and $G_{k}^{\prime}\left(\beta_{0}\right) \geq 0, I_{k}^{\prime}\left(\beta_{0}\right) \leq 0$, and for $x \geq y$,

$$
I_{k}^{\prime}(x)-I_{k}^{\prime}(y) \leq L_{k}\|x-y\|, \quad G_{k}^{\prime}(y)-G_{k}^{\prime}(x) \leq M_{k}\|x-y\|,
$$

where $L_{k}>0, M_{k}>0, k=1,2, \ldots, p$, are constant matrices.

(4) The function $g_{x}\left(t, \beta_{0}(t)\right) x$ is quasimonotone nondecreasing in $x$ and the function $\left(g_{x}\left(t, \alpha_{0}\right)-f_{x}\left(t, \alpha_{0}\right)\right) e @ x$ is strictly decreasing in $x$ on $[0, T]$.

(5) The inequality $\left(G_{k}^{\prime}\left(\beta_{0}\left(\tau_{k}\right)\right)-I_{k}^{\prime}\left(\beta_{0}\left(\tau_{k}\right)\right)\right) e \leq e$ holds.

Then there exist two sequences of functions $\left\{\alpha_{m}(t)\right\}_{0}^{\infty}$ and $\left\{\beta_{m}(t)\right\}_{0}^{\infty}$ such that

(a) the sequences are increasing and decreasing correspondingly;

(b) both sequences uniformly converge on the intervals $\left(\tau_{k}, \tau_{k+1}\right]$ to the unique solution of the PBVP (2.1)-(2.2) in $S\left(\alpha_{0}, \beta_{0}\right), k=0,1,2, \ldots, p$;

(c) the convergence is quadratic.

Proof. Consider the periodic boundary value problem for the system of impulsive linear differential equations

$$
\begin{gathered}
x^{\prime}(t)=f\left(t, \beta_{0}(t)\right)+g\left(t, \alpha_{0}(t)\right)+g_{x}\left(t, \beta_{0}\right)\left(x-\alpha_{0}\right) \\
\quad+f_{x}\left(t, \beta_{0}\right)\left(y-\beta_{0}\right) \quad \text { for } t \in[0, T], t \neq \tau_{k}, \\
\begin{aligned}
& y^{\prime}(t)=f\left(t, \alpha_{0}(t)\right)+g\left(t, \beta_{0}(t)\right)+g_{x}\left(t, \beta_{0}\right)\left(y-\beta_{0}\right)+f_{x}\left(t, \beta_{0}\right)\left(x-\alpha_{0}\right), \\
& x\left(\tau_{k}+0\right)= I_{k}\left(\beta_{0}\left(\tau_{k}\right)\right)+G_{k}\left(\alpha_{0}\left(\tau_{k}\right)\right)+G_{k}^{\prime}\left(\beta_{0}\left(\tau_{k}\right)\right)\left[x\left(\tau_{k}\right)-\alpha_{0}\left(\tau_{k}\right)\right] \\
&+I_{k}^{\prime}\left(\beta_{0}\left(\tau_{k}\right)\right)\left[y\left(\tau_{k}\right)-\beta_{0}\left(\tau_{k}\right)\right], \\
& y\left(\tau_{k}+0\right)= I_{k}\left(\alpha_{0}\left(\tau_{k}\right)\right)+G_{k}\left(\beta_{0}\left(\tau_{k}\right)\right)+G_{k}^{\prime}\left(\beta_{0}\left(\tau_{k}\right)\right)\left[y\left(\tau_{k}\right)-\beta_{0}\left(\tau_{k}\right)\right] \\
&+I_{k}^{\prime}\left(\beta_{0}\left(\tau_{k}\right)\right)\left[x\left(\tau_{k}\right)-\alpha_{0}\left(\tau_{k}\right)\right], \\
& x(0)=x(T), \quad y(0)=y(T) .
\end{aligned}
\end{gathered}
$$

The PBVP (3.46) can be written in the form

$$
\begin{gathered}
p^{\prime}=A^{(0)}(t) p(t)+h^{(0)}(t) \quad \text { for } t \in[0, T], t \neq \tau_{k}, \\
p\left(\tau_{k}+0\right)=B_{k}^{(0)} p\left(\tau_{k}\right)+\sigma_{k}^{(0)}, \quad p(0)=p(T),
\end{gathered}
$$


where

$$
\begin{gathered}
p=\left(\begin{array}{l}
x \\
y
\end{array}\right), \quad A^{(0)}(t)=\left(\begin{array}{ll}
g_{x}\left(t, \beta_{0}\right) & f_{x}\left(t, \beta_{0}\right) \\
f_{x}\left(t, \beta_{0}\right) & g_{x}\left(t, \beta_{0}\right)
\end{array}\right), \quad B_{k}^{(0)}=\left(\begin{array}{cc}
G_{k}^{\prime}\left(\beta_{0}\left(\tau_{k}\right)\right) & I_{k}^{\prime}\left(\beta_{0}\left(\tau_{k}\right)\right) \\
I_{k}^{\prime}\left(\beta_{0}\left(\tau_{k}\right)\right) & G_{k}^{\prime}\left(\beta_{0}\left(\tau_{k}\right)\right)
\end{array}\right), \\
h^{(0)}(t)=\left(\begin{array}{l}
f\left(t, \beta_{0}(t)\right)+g\left(t, \alpha_{0}(t)\right)-g_{x}\left(t, \beta_{0}\right) \alpha_{0}-f_{x}\left(t, \beta_{0}\right) \beta_{0} \\
f\left(t, \alpha_{0}(t)\right)+g\left(t, \beta_{0}(t)\right)-g_{x}\left(t, \beta_{0}\right) \beta_{0}-f_{x}\left(t, \beta_{0}\right) \alpha_{0}
\end{array}\right), \\
\sigma_{k}^{(0)}=\left(\begin{array}{l}
I_{k}\left(\beta_{0}\left(\tau_{k}\right)\right)+G_{k}\left(\alpha_{0}\left(\tau_{k}\right)\right)-G_{k}^{\prime}\left(\beta_{0}\left(\tau_{k}\right)\right) \alpha_{0}\left(\tau_{k}\right)-I_{k}^{\prime}\left(\beta_{0}\left(\tau_{k}\right)\right) \beta_{0}\left(\tau_{k}\right) \\
G_{k}\left(\alpha_{0}\left(\tau_{k}\right)\right)+I_{k}\left(\beta_{0}\left(\tau_{k}\right)\right)-G_{k}^{\prime}\left(\beta_{0}\left(\tau_{k}\right)\right) \beta_{0}\left(\tau_{k}\right)-I_{k}^{\prime}\left(\beta_{0}\left(\tau_{k}\right)\right) \alpha_{0}\left(\tau_{k}\right)
\end{array}\right) .
\end{gathered}
$$

Consider the matrices

$$
C^{0}(t)=\left(\begin{array}{cc}
g_{x}\left(t, \beta_{0}\right) & -f_{x}\left(t, \beta_{0}\right) \\
-f_{x}\left(t, \beta_{0}\right) & g_{x}\left(t, \beta_{0}\right)
\end{array}\right), \quad D_{k}^{0}=\left(\begin{array}{cc}
G_{k}^{\prime}\left(\beta_{0}\left(\tau_{k}\right)\right) & -I_{k}^{\prime}\left(\beta_{0}\left(\tau_{k}\right)\right) \\
-I_{k}^{\prime}\left(\beta_{0}\left(\tau_{k}\right)\right) & G_{k}^{\prime}\left(\beta_{0}\left(\tau_{k}\right)\right)
\end{array}\right) .
$$

From conditions (2), (3), (4), and (5) of Theorem 3.2, it follows that $C^{0}(t) \in \Xi$ and $D_{k}^{0} \in \Psi$. According to Lemma 2.8, $\operatorname{det}\left(E-C^{0}(t)\right) \neq 0$. Therefore, $\operatorname{det}\left(E-A^{0}(t)\right)=\operatorname{det}(E-$ $\left.C^{0}(t)\right) \neq 0$. Therefore, according to Lemma 2.9, the boundary value problem (3.46) has a unique solution $\alpha_{1}(t), \beta_{1}(t)$.

We will prove that $\alpha_{0}(t) \leq \alpha_{1}(t)$ and $\beta_{0}(t) \geq \beta_{1}(t)$ on $[0, T]$. Set $p(t)=\alpha_{0}(t)-\alpha_{1}(t)$, $q(t)=\beta_{1}(t)-\beta_{0}(t)$. Then we have

$$
\begin{gathered}
p^{\prime} \leq-f_{x}\left(t, \beta_{0}\right) q+g_{x}\left(t, \beta_{0}\right) p, \\
q^{\prime} \leq g_{x}\left(t, \beta_{0}\right) q-f_{x}\left(t, \beta_{0}\right) p \quad \text { for } t \in[0, T], t \neq \tau_{k}, \\
p\left(\tau_{k}+0\right) \leq G_{k}^{\prime}\left(\beta_{0}\left(\tau_{k}\right)\right) p\left(\tau_{k}\right)-I_{k}^{\prime}\left(\beta_{0}\left(\tau_{k}\right)\right) q\left(\tau_{k}\right), \\
q\left(\tau_{k}+0\right) \leq G_{k}^{\prime}\left(\beta_{0}\left(\tau_{k}\right)\right) q\left(\tau_{k}\right)-I_{k}^{\prime}\left(\beta_{0}\left(\tau_{k}\right)\right) p\left(\tau_{k}\right), \\
p(0) \leq p(T), \quad q(0) \leq q(T) .
\end{gathered}
$$

The PBVP (3.50) can be written in the form

$$
\begin{gathered}
m^{\prime}(t) \leq A(t) m(t) \quad \text { for } t \in[0, T], t \neq \tau_{k} \\
m\left(\tau_{k}+0\right) \leq B_{k} m\left(\tau_{k}\right) \\
m(0) \leq m(T)
\end{gathered}
$$

where

$$
m=\left(\begin{array}{l}
p \\
q
\end{array}\right), \quad A(t)=\left(\begin{array}{cc}
g_{x}\left(t, \beta_{0}\right) & -f_{x}\left(t, \beta_{0}\right) \\
-f_{x}\left(t, \beta_{0}\right) & g_{x}\left(t, \beta_{0}\right)
\end{array}\right), \quad B_{k}=\left(\begin{array}{cc}
G_{k}^{\prime}\left(\beta_{0}\left(\tau_{k}\right)\right) & -I_{k}^{\prime}\left(\beta_{0}\left(\tau_{k}\right)\right) \\
-I_{k}^{\prime}\left(\beta_{0}\left(\tau_{k}\right)\right) & G_{k}^{\prime}\left(\beta_{0}\left(\tau_{k}\right)\right)
\end{array}\right) .
$$


According to Lemma 2.6, $m(t) \leq 0$ on $[0, T]$, that is, $\alpha_{0}(t) \leq \alpha_{1}(t)$ and $\beta_{1}(t) \leq \beta_{0}(t)$ on $[0, T]$.

Following the same ideas as in the proof of Theorem 3.1, we can construct two sequences of functions $\left\{\alpha_{m}(t)\right\}_{0}^{\infty}$ and $\left\{\beta_{m}(t)\right\}_{0}^{\infty}, \alpha_{m}, \beta_{m} \in S\left(\alpha_{m-1}, \beta_{m-1}\right)$ where the functions are the unique solution of the boundary value problem for the linear system of impulsive differential equations

$$
\begin{gathered}
x^{\prime}(t)=f\left(t, \beta_{m}(t)\right)+g\left(t, \alpha_{m}(t)\right)+g_{x}\left(t, \beta_{m}\right)\left(x-\alpha_{m}\right) \\
+f_{x}\left(t, \beta_{m}\right)\left(y-\beta_{m}\right) \quad \text { for } t \in[0, T], t \neq \tau_{k}, \\
y^{\prime}(t)=f\left(t, \alpha_{m}(t)\right)+g\left(t, \beta_{m}(t)\right)+g_{x}\left(t, \beta_{m}\right)\left(y-\beta_{m}\right)+f_{x}\left(t, \beta_{m}\right)\left(x-\alpha_{m}\right), \\
x\left(\tau_{k}+0\right)=I_{k}\left(\beta_{m}\left(\tau_{k}\right)\right)+G_{k}\left(\alpha_{m}\left(\tau_{k}\right)\right)+G_{k}^{\prime}\left(\beta_{m}\left(\tau_{k}\right)\right)\left[x\left(\tau_{k}\right)-\alpha_{m}\left(\tau_{k}\right)\right] \\
+I_{k}^{\prime}\left(\beta_{m}\left(\tau_{k}\right)\right)\left[y\left(\tau_{k}\right)-\beta_{m}\left(\tau_{k}\right)\right], \\
y\left(\tau_{k}+0\right)=I_{k}\left(\alpha_{m}\left(\tau_{k}\right)\right)+G_{k}\left(\beta_{m}\left(\tau_{k}\right)\right)+G_{k}^{\prime}\left(\beta_{m}\left(\tau_{k}\right)\right)\left[y\left(\tau_{k}\right)-\beta_{m}\left(\tau_{k}\right)\right] \\
+I_{k}^{\prime}\left(\beta_{m}\left(\tau_{k}\right)\right)\left[x\left(\tau_{k}\right)-\alpha_{m}\left(\tau_{k}\right)\right], \\
x(0)=x(T), \quad y(0)=y(T) .
\end{gathered}
$$

The inequality $\alpha_{m}(t) \leq \beta_{m}(t), t \in[0, T]$, holds and both sequences are uniformly convergent.

Denote

$$
\lim _{m \rightarrow \infty} \alpha_{m}(t)=u(t), \quad \lim _{m \rightarrow \infty} \beta_{m}(t)=v(t)
$$

From the uniform convergence and the definition of the functions $\alpha_{m}(t)$ and $\beta_{m}(t)$, the validity of the inequalities

$$
\alpha_{0}(t) \leq u(t) \leq v(t) \leq \beta_{0}(t)
$$

follows.

Since the functions $\alpha_{m}(t)$ and $\beta_{m}(t)$ are solutions of the PBVP (3.53), we obtain that the functions $u(t)$ and $v(t)$ are solutions of the PBVP

$$
\begin{gathered}
u^{\prime}=f(t, v(t))+g(t, u(t)), \quad v^{\prime}=f(t, u(t))+g(t, v(t)) \quad \text { for } t \in[0, T], t \neq \tau_{k}, \\
u\left(\tau_{k}+0\right)=I_{k}\left(v\left(\tau_{k}\right)\right)+G_{k}\left(u\left(\tau_{k}\right)\right), \\
v\left(\tau_{k}+0\right)=I_{k}\left(u\left(\tau_{k}\right)\right)+G_{k}\left(v\left(\tau_{k}\right)\right), \\
u(0)=u(T), \quad v(0)=v(T) .
\end{gathered}
$$

As in the proof of Theorem 3.1, we can obtain that $u(t)=v(t)$ on $[0, T]$.

We will prove that the convergence is quadratic. 
Define the functions $p_{m+1}(t)=u(t)-\alpha_{m+1}(t)$ and $q_{m+1}(t)=\beta_{m+1}(t)-u(t), t \in[0, T]$. For $t \in[0, T], t \neq \tau_{k}$, we obtain the inequalities

$$
\begin{aligned}
p_{m+1}^{\prime} \leq & f(t, u)-f\left(t, \beta_{m}\right)+g(t, u)-g\left(t, \alpha_{m}\right)+f_{x}\left(t, \beta_{m}\right)\left(q_{m}-q_{m+1}\right)+g_{x}\left(t, \beta_{m}\right)\left(p_{m+1}-p_{m}\right) \\
= & -\left(\int_{0}^{1} f_{x}\left(t, \lambda u+(1-\lambda) \beta_{m}\right) d \lambda\right) q_{m}+\left(\int_{0}^{1} g_{x}\left(t, \lambda u+(1-\lambda) \alpha_{m}\right) d \lambda\right) p_{m} \\
& +f_{x}\left(t, \beta_{m}\right)\left(q_{m}-q_{m+1}\right)+g_{x}\left(t, \beta_{m}\right)\left(p_{m+1}-p_{m}\right) \\
\leq & \left(f_{x}\left(t, \beta_{m}\right)-f_{x}(t, u)\right) q_{m}+\left(g_{x}\left(t, \alpha_{m}\right)-g_{x}\left(t, \beta_{m}\right)\right) p_{m} \\
& -f_{x}\left(t, \beta_{m}\right) q_{m+1}+g_{x}\left(t, \beta_{m}\right) p_{m+1} \\
\leq & g_{x}\left(t, \beta_{m}\right) p_{m+1}-f_{x}\left(t, \beta_{m}\right) q_{m+1}+S_{1}\left\|q_{m}\right\| q_{m}+S_{2}\left\|\alpha_{m}-\beta_{m}\right\| p_{m} \\
\leq & g_{x}\left(t, \beta_{m}\right) p_{m+1}-f_{x}\left(t, \beta_{m}\right) q_{m+1}+\left(\tilde{s}+\frac{1}{2} \tilde{m}\right)\left\|q_{m}\right\|^{2}+\frac{3}{2} \tilde{m}\left\|p_{m}\right\|^{2},
\end{aligned}
$$

where $\widetilde{s}=\left(\widetilde{s_{1}}, \widetilde{s_{2}}, \ldots, \widetilde{s_{m}}\right), \widetilde{m}=\left(\widetilde{m_{1}}, \widetilde{m_{2}}, \ldots, \widetilde{m_{n}}\right), \widetilde{s_{i}}=\sum_{j=1}^{n} S_{i j}^{1}, \widetilde{m_{i}}=\sum_{j=1}^{n} S_{i j}^{2}, i=1,2, \ldots, n$.

For $t \in[0, T], t \neq \tau_{k}$, from the definition of the functions $\alpha_{m}(t)$ and $\beta_{m}(t)$ and the fact that $u(t)$ is a solution of the PBVP (2.1)-(2.2), we obtain

$$
\begin{aligned}
q_{n+1}^{\prime} & \leq-f_{x}\left(t, \beta_{m}\right) p_{m+1}+g_{x}\left(t, \beta_{m}\right) q_{m+1}+S_{1}\left\|q_{m}+p_{m}\right\| p_{m}+S_{2}\left\|q_{m}\right\| q_{m} \\
& \leq-f_{x}\left(t, \beta_{m}\right) p_{m+1}+g_{x}\left(t, \beta_{m}\right) q_{m+1}+\frac{3}{2} \widetilde{s}\left\|p_{m}\right\|^{2}+\left(\tilde{m}+\frac{1}{2} \widetilde{s}\right)\left\|q_{m}\right\|^{2} .
\end{aligned}
$$

For $k=1,2, \ldots, p$, from the jump conditions, we have

$$
\begin{aligned}
p_{m+1}\left(\tau_{k}+0\right) \leq & I_{k}\left(u\left(\tau_{k}\right)\right)-I_{k}\left(\alpha_{m}\left(\tau_{k}\right)\right)+G_{k}\left(u\left(\tau_{k}\right)\right) \\
& -G_{k}\left(\alpha_{m}\left(\tau_{k}\right)\right) I_{k}^{\prime}\left(\alpha_{m}\left(\tau_{k}\right)\right)\left[\alpha_{m+1}\left(\tau_{k}\right)-\alpha_{m}\left(\tau_{k}\right)\right] \\
& -G_{k}\left(\alpha_{m}\left(\tau_{k}\right)\right)\left[\beta_{m+1}\left(\tau_{k}\right)-\beta_{m}\left(\tau_{k}\right)\right] \\
\leq & -G_{k}\left(\alpha_{m}\left(\tau_{k}\right)\right) p_{m+1}\left(\tau_{k}\right)+I_{k}^{\prime}\left(\alpha_{m}\left(\tau_{k}\right)\right) q_{m+1}\left(\tau_{k}\right) \\
& +\mu_{1}\left\|p_{m}\left(\tau_{k}\right)\right\|^{2}+\mu_{2}\left\|q_{m}\left(\tau_{k}\right)\right\|^{2}, \\
q_{m+1}\left(\tau_{k}+0\right) \leq & I_{k}^{\prime}\left(\alpha_{m}\left(\tau_{k}\right)\right) p_{m+1}\left(\tau_{k}\right)-G_{k}\left(\alpha_{m}\left(\tau_{k}\right)\right) q_{m+1}\left(\tau_{k}\right) \\
& +\widetilde{\mu_{1}}\left\|p_{m}\left(\tau_{k}\right)\right\|^{2}+\widetilde{\mu_{2}}\left\|q_{m}\left(\tau_{k}\right)\right\|^{2},
\end{aligned}
$$

where $\mu_{i}, \tilde{\mu_{i}}, i=1,2$ are constant vectors.

The differential inequalities (3.57)-(3.60) and the periodic conditions for the function $p(t)$ and $q(t)$ can be written in the form

$$
\begin{gathered}
r_{m+1}^{\prime} \leq A(t) r_{m+1}+P\left\|r_{m}\right\|^{2}, \quad t \neq \tau_{k}, \\
r_{m+1}\left(\tau_{k}+0\right) \leq B_{k} r_{m+1}\left(\tau_{k}\right)+Q_{k}\left\|r_{m}\left(\tau_{k}\right)\right\|^{2}, \\
r_{m+1}(0)=r_{m+1}(T),
\end{gathered}
$$


where

$$
\begin{gathered}
r_{m+1}=\left(\begin{array}{c}
p_{m+1} \\
q_{m+1}
\end{array}\right), \quad P=\left(\begin{array}{c}
\tilde{s}+2 \tilde{m} \\
2 \tilde{s}+\tilde{m}
\end{array}\right), \quad Q_{k}=\left(\begin{array}{c}
\mu_{1}+\mu_{2} \\
\widetilde{\mu_{1}}+\widetilde{\mu_{2}}
\end{array}\right), \\
A(t)=\left(\begin{array}{cc}
g_{x}\left(t, \beta_{m}\right) & -f_{x}\left(t, \beta_{m}\right) \\
-f_{x}\left(t, \beta_{m}\right) & g_{x}\left(t, \beta_{m}\right)
\end{array}\right), \quad B_{k}=\left(\begin{array}{cc}
G_{k}^{\prime}\left(\beta_{m}\left(\tau_{k}\right)\right) & -I_{k}^{\prime}\left(\beta_{m}\left(\tau_{k}\right)\right) \\
-I_{k}^{\prime}\left(\beta_{m}\left(\tau_{k}\right)\right) & G_{k}^{\prime}\left(\beta_{m}\left(\tau_{k}\right)\right)
\end{array}\right) .
\end{gathered}
$$

According to Lemma 2.10, the inequality

$$
r_{m+1}(t) \leq W(t, 0) x_{0}+\int_{0}^{t} W(t, s) P\left\|r_{m}(s)\right\|^{2} d s+\sum_{0<t_{k}<t} W\left(t, t_{k}+0\right) Q_{k}\left\|r_{m}\left(\tau_{k}\right)\right\|^{2}
$$

holds for $t \in[0, T]$, where

$$
x_{0}=(E-W(T, 0))^{-1}\left(\int_{0}^{T} W(T, s) P\left\|r_{m}(s)\right\|^{2} d s+\sum_{k=1}^{p} W\left(T, t_{k}+0\right) Q_{k}\left\|r_{m}\left(\tau_{k}\right)\right\|^{2}\right)
$$

and $W(t, s)$ is defined by $(2.14)$.

From the inequalities (3.63), (3.64), it follows that there exists a number $\lambda>0$ such that $\left\|\left|r_{m+1}\right|\right\||\leq \lambda| \| r_{m}||^{2}$, where $\||r|\|=\sup _{t \in[0, T]}\|r(t)\|$. This inequality proves the quadratic convergence.

The next theorem is about the case when the PBVP (2.1)-(2.2) has a lower solution as well as an upper solution.

Theorem 3.3. Let the following conditions hold.

(1) The functions $\alpha_{0}(t), \beta_{0}(t) \in P C^{1}\left([0, T], \mathbb{R}^{n}\right), \alpha_{0}(t) \leq \beta_{0}(t)$ for $t \in[0, T]$, are natural lower and upper solutions of the PBVP (2.1), (2.2).

(2) The functions $f_{x}, g_{x}$ exist and are continuous on $\Omega\left(\alpha_{0}, \beta_{0}\right), f_{x}(t, x)$ is nondecreasing in $x, g_{x}(t, x)$ is nonincreasing in $x$ for $t \in[0, T]$, and for $x \geq y$,

$$
f_{x}(t, x)-f_{x}(t, y) \leq S_{1}\|x-y\|, \quad g_{x}(t, y)-g_{x}(t, x) \leq S_{2}\|x-y\|,
$$

where $S_{1}>0, S_{2}>0$ are constant matrices.

(3) The functions $I_{k}, G_{k} \in C^{1}\left(D_{k}\left(\alpha_{0}, \beta_{0}\right), \mathbb{R}^{n}\right), I_{k}^{\prime}(x)$ are nondecreasing, $G_{k}^{\prime}(x)$ are nonincreasing, $k=1,2, \ldots, p$, and $I_{k}^{\prime}\left(\alpha_{0}\left(\tau_{k}\right)\right)+G_{k}^{\prime}\left(\beta_{0}\left(\tau_{k}\right)\right) \geq 0$ and for $x \geq y$,

$$
I_{k}^{\prime}(x)-I_{k}^{\prime}(y) \leq L_{k}\|x-y\|, \quad G_{k}^{\prime}(y)-G_{k}^{\prime}(x) \leq M_{k}\|x-y\|,
$$

where $L_{k}>0, M_{k}>0, k=1,2, \ldots, p$, are constant matrices. 
(4) The function $\left(f_{x}\left(t, \alpha_{0}(t)\right)+g_{x}\left(t, \beta_{0}(t)\right)\right) x$ is quasimonotone nondecreasing in $x$ and the function $\left(f_{x}\left(t, \beta_{0}\right)+g_{x}\left(t, \alpha_{0}\right)\right) e @ x$ is strictly decreasing in $x$ on $[0, T]$.

(5) The inequality $\left(I_{k}^{\prime}\left(\beta_{0}\left(\tau_{k}\right)\right)+G_{k}^{\prime}\left(\alpha_{0}\left(\tau_{k}\right)\right)\right) e \leq e$ holds.

Then there exist two sequences of functions $\left\{\alpha_{m}(t)\right\}_{0}^{\infty}$ and $\left\{\beta_{m}(t)\right\}_{0}^{\infty}$ such that

(a) the sequences are increasing and decreasing correspondingly;

(b) both sequences uniformly converge on the intervals $\left(\tau_{k}, \tau_{k+1}\right]$ to the unique solution of the PBVP (2.1)-(2.2) in $S\left(\alpha_{0}, \beta_{0}\right), k=0,1,2, \ldots, p$;

(c) the convergence is quadratic.

Proof. Consider the periodic boundary value problem for the system of impulsive differential equations

$$
\begin{gathered}
x^{\prime}(t)=f\left(t, \alpha_{0}(t)\right)+g\left(t, \alpha_{0}(t)\right)+\left(f_{x}\left(t, \alpha_{0}\right)+g_{x}\left(t, \beta_{0}\right)\right)\left(x-\alpha_{0}\right), \quad t \neq \tau_{k}, \\
x\left(\tau_{k}+0\right)=I_{k}\left(\alpha_{0}\left(\tau_{k}\right)\right)+G_{k}\left(\alpha_{0}\left(\tau_{k}\right)\right)+\left(I_{k}^{\prime}\left(\alpha_{0}\left(\tau_{k}\right)\right)+G_{k}^{\prime}\left(\beta_{0}\left(\tau_{k}\right)\right)\right)\left[x\left(\tau_{k}\right)-\alpha_{0}\left(\tau_{k}\right)\right], \\
x(0)=x(T) .
\end{gathered}
$$

From conditions (2), (3), (4), and (5) of Theorem 3.3, it follows that the matrices $A(t)=$ $f_{x}\left(t, \alpha_{0}\right)+g_{x}\left(t, \beta_{0}\right) \in \Xi$ and $B_{k}=I_{k}^{\prime}\left(\alpha_{0}\left(\tau_{k}\right)\right)+G_{k}^{\prime}\left(\beta_{0}\left(\tau_{k}\right)\right) \in \Psi$. According to Lemmas 2.8 and 2.9, the PBVP (3.67) has a unique solution $\alpha_{1}(t)$.

Consider the periodic boundary value problem for the system of impulsive differential equations

$$
\begin{aligned}
& y^{\prime}(t)=f\left(t, \beta_{0}(t)\right)+g\left(t, \beta_{0}(t)\right)+\left(f_{x}\left(t, \alpha_{0}\right)+g_{x}\left(t, \beta_{0}\right)\right)\left(y-\beta_{0}\right), \quad t \neq \tau_{k}, \\
& \begin{aligned}
& y\left(\tau_{k}+0\right)= I_{k}\left(\beta_{0}\left(\tau_{k}\right)\right)+G_{k}\left(\beta_{0}\left(\tau_{k}\right)\right) \\
& \quad+\left(I_{k}^{\prime}\left(\alpha_{0}\left(\tau_{k}\right)\right)+G_{k}^{\prime}\left(\beta_{0}\left(\tau_{k}\right)\right)\right)\left[y\left(\tau_{k}\right)-\alpha_{0}\left(\tau_{k}\right)\right], \\
& y(0)=y(T) .
\end{aligned}
\end{aligned}
$$

According to Lemmas 2.8 and 2.9, the PBVP (3.68)-(3.70) has a unique solution $\beta_{1}(t)$.

We will prove that $\alpha_{0}(t) \leq \alpha_{1}(t)$ and $\beta_{0}(t) \geq \beta_{1}(t)$ on $[0, T]$. Set $p(t)=\alpha_{0}(t)-\alpha_{1}(t)$, $q(t)=\beta_{1}(t)-\beta_{0}(t)$. The functions $p(t)$ and $q(t)$ satisfy the following impulsive inequalities:

$$
\begin{gathered}
m^{\prime} \leq\left(f_{x}\left(t, \alpha_{0}\right)+g_{x}\left(t, \beta_{0}\right)\right) m \text { for } t \in[0, T], t \neq \tau_{k}, \\
m\left(\tau_{k}+0\right) \leq\left(I_{k}^{\prime}\left(\alpha_{0}\left(\tau_{k}\right)\right)+G_{k}^{\prime}\left(\beta_{0}\left(\tau_{k}\right)\right)\right) m\left(\tau_{k}\right), \\
m(0) \leq m(T) .
\end{gathered}
$$

According to Lemma 2.6, $m(t) \leq 0$ and $p(t) \leq 0$ and $q(t) \leq 0$ on $[0, T]$, that is, $\alpha_{0}(t) \leq$ $\alpha_{1}(t)$ and $\beta_{1}(t) \leq \beta_{0}(t)$ on $[0, T]$.

Consider the function $p(t)=\alpha_{1}(t)-\beta_{1}(t)$. Then from the PBVP (3.67)-(3.70), conditions (2), (3), (4), and (5) of Theorem 3.3, and Lemma 2.11, we obtain that the function $p(t)$ satisfies the inequalities (3.71). According to Lemma 2.6, we have $p(t) \leq 0$ on $[0, T]$, that is, $\alpha_{1}(t) \leq \beta_{1}(t)$. 
As in the proof of Theorem 3.1, we can obtain both sequences of functions $\left\{\alpha_{m}(t)\right\}$ and $\left\{\beta_{m}(t)\right\}$, which are the unique solutions of the PBVPs

$$
\begin{aligned}
x^{\prime}(t)= & f\left(t, \alpha_{m-1}(t)\right)+g\left(t, \alpha_{m-1}(t)\right) \\
& +\left(f_{x}\left(t, \alpha_{m-1}\right)+g_{x}\left(t, \beta_{m-1}\right)\right)\left(x-\alpha_{m-1}\right) \quad \text { for } t \in[0, T], t \neq \tau_{k}, \\
x\left(\tau_{k}+0\right)= & I_{k}\left(\alpha_{m-1}\left(\tau_{k}\right)\right)+G_{k}\left(\alpha_{m-1}\left(\tau_{k}\right)\right) \\
& +\left(I_{k}^{\prime}\left(\alpha_{m-1}\left(\tau_{k}\right)\right)+G_{k}^{\prime}\left(\beta_{m-1}\left(\tau_{k}\right)\right)\right)\left[x\left(\tau_{k}\right)-\alpha_{m-1}\left(\tau_{k}\right)\right], \\
& x(0)=x(T) ; \\
y^{\prime}(t)= & f\left(t, \beta_{m-1}(t)\right)+g\left(t, \beta_{m-1}(t)\right) \\
& +\left(f_{x}\left(t, \alpha_{m-1}\right)+g_{x}\left(t, \beta_{m-1}\right)\right)\left(y-\beta_{m-1}\right), \quad \text { for } t \in[0, T], t \neq \tau_{k}, \\
y\left(\tau_{k}+0\right)= & I_{k}\left(\beta_{m-1}\left(\tau_{k}\right)\right)+G_{k}\left(\beta_{m-1}\left(\tau_{k}\right)\right) \\
& +\left(I_{k}^{\prime}\left(\alpha_{m-1}\left(\tau_{k}\right)\right)+G_{k}^{\prime}\left(\beta_{m-1}\left(\tau_{k}\right)\right)\left[y\left(\tau_{k}\right)-\beta_{m-1}\left(\tau_{k}\right)\right],\right. \\
& y(0)=y(T) .
\end{aligned}
$$

We can prove that $\alpha_{m}, \beta_{m} \in S\left(\alpha_{m-1}, \beta_{m-1}\right), \alpha_{m}(t) \leq \beta_{m}(t), t \in[0, T]$, and both sequences are convergent.

Let their limits be $u(t)$ and $v(t)$ correspondingly.

The functions $u(t), v(t)$ satisfy the inequalities

$$
\alpha_{0}(t) \leq u(t) \leq v(t) \leq \beta_{0}(t)
$$

and are solutions of the PBVP (2.1)-(2.2). Since the functions $f, g$ and $I_{k}, G_{k}$ are Lipschitz, it follows that $u(t)=v(t)$ on $[0, T]$.

We will prove that the convergence is quadratic.

Define the functions $p_{m+1}(t)=u(t)-\alpha_{m+1}(t)$ and $q_{m+1}(t)=\beta_{m+1}(t)-u(t), t \in[0, T]$. For $t \in[0, T], t \neq \tau_{k}$, we obtain the inequalities

$$
\begin{aligned}
p_{m+1}^{\prime} \leq & f(t, u)-f\left(t, \alpha_{m}\right)+g(t, u)-g\left(t, \alpha_{m}\right)+\left(f_{x}\left(t, \alpha_{m}\right)+g_{x}\left(t, \beta_{m}\right)\right)\left(p_{m+1}-p_{m}\right) \\
= & \left(\int_{0}^{1} f_{x}\left(t, \lambda u+(1-\lambda) \alpha_{m}\right) d \lambda\right) p_{m}+\left(\int_{0}^{1} g_{x}\left(t, \lambda u+(1-\lambda) \alpha_{m}\right) d \lambda\right) p_{m} \\
& +\left(f_{x}\left(t, \alpha_{m}\right)+g_{x}\left(t, \beta_{m}\right)\right)\left(p_{m+1}-p_{m}\right) \\
\leq & \left(f_{x}(t, u)-f_{x}\left(t, \alpha_{m}\right)\right) p_{m}+\left(g_{x}\left(t, \alpha_{m}\right)-g_{x}\left(t, \beta_{m}\right)\right) p_{m} \\
& +\left(f_{x}\left(t, \alpha_{m}\right)+g_{x}\left(t, \beta_{m}\right)\right) p_{m+1} \\
\leq & \left(f_{x}\left(t, \alpha_{m}\right)+g_{x}\left(t, \beta_{m}\right)\right) p_{m+1}+S_{1}\left\|p_{m}\right\| p_{m}+S_{2}\left\|\alpha_{m}-\beta_{m}\right\| p_{m} \\
\leq & \left(f_{x}\left(t, \alpha_{m}\right)+g_{x}\left(t, \beta_{m}\right)\right) p_{m+1}+\left(\tilde{s}+\frac{1}{2} \tilde{m}\right)\left\|p_{m}\right\|^{2}+\frac{1}{2} \tilde{m}\left\|q_{m}\right\|^{2},
\end{aligned}
$$

where $\tilde{s}=\left(\widetilde{s_{1}}, \widetilde{s_{2}}, \ldots, \widetilde{s_{m}}\right), \tilde{m}=\left(\widetilde{m_{1}}, \widetilde{m_{2}}, \ldots, \widetilde{m_{m}}\right), \widetilde{s_{i}}=\sum_{j=1}^{n} S_{i j}^{(1)}, \widetilde{m_{i}}=\sum_{j=1}^{n} S_{i j}^{(2)}, i=1,2, \ldots, n$. 
For $t \in[0, T], t \neq \tau_{k}$, from the definition of the functions $\alpha_{m}(t)$ and $\beta_{m}(t)$ and the fact that $u(t)$ is a solution of the PBVP (2.1)-(2.2), we obtain

$$
\begin{aligned}
q_{m+1}^{\prime} \leq & f\left(t, \beta_{m}\right)-f(t, u)+g\left(t, \beta_{m}\right)-g(t, u)+\left(f_{x}\left(t, \alpha_{m}\right)+g_{x}\left(t, \beta_{m}\right)\right)\left(q_{m+1}-q_{m}\right) \\
= & \left(\int_{0}^{1} f_{x}\left(t, \lambda \beta_{m}+(1-\lambda) u\right) d \lambda\right) q_{m}+\left(\int_{0}^{1} g_{x}\left(t, \lambda \beta_{m}+(1-\lambda) u\right) d \lambda\right) q_{m} \\
& +\left(f_{x}\left(t, \alpha_{m}\right)+g_{x}\left(t, \beta_{m}\right)\right)\left(q_{m+1}-q_{m}\right) \leq\left(f_{x}\left(t, \beta_{m}\right)-f_{x}\left(t, \alpha_{m}\right)\right) q_{m} \\
& +\left(g_{x}(t, u)-g_{x}\left(t, \beta_{m}\right)\right) q_{m}+\left(f_{x}\left(t, \beta_{m}\right)+g_{x}\left(t, \beta_{m}\right)\right) q_{m+1} \\
\leq & \left(f_{x}\left(t, \alpha_{m}\right)+g_{x}\left(t, \beta_{m}\right)\right) q_{m+1}+S_{1}\left\|\alpha_{m}-\beta_{m}\right\| q_{m}+S_{2}\left\|q_{m}\right\| q_{m} \\
\leq & \left(f_{x}\left(t, \alpha_{m}\right)+g_{x}\left(t, \beta_{m}\right)\right) q_{m+1}+\frac{1}{2} \tilde{s}\left\|p_{m}\right\|^{2}+\left(\frac{3}{2} \tilde{s}+\tilde{m}\right)\left\|q_{m}\right\|^{2} .
\end{aligned}
$$

For $k=1,2, \ldots, p$, from the jump conditions, we have

$$
\begin{aligned}
p_{m+1}\left(\tau_{k}+0\right) \leq & I_{k}\left(u\left(\tau_{k}\right)\right)-I_{k}\left(\alpha_{m}\left(\tau_{k}\right)\right)+G_{k}\left(u\left(\tau_{k}\right)\right)-G_{k}\left(\alpha_{m}\left(\tau_{k}\right)\right) \\
& +\left(I_{k}^{\prime}\left(\alpha_{m}\left(\tau_{k}\right)\right)+G_{k}\left(\beta_{m}\left(\tau_{k}\right)\right)\right)\left[p_{m+1}\left(\tau_{k}\right)-p_{m}\left(\tau_{k}\right)\right] \\
\leq & \left(I_{k}^{\prime}\left(\alpha_{m}\left(\tau_{k}\right)\right)+G_{k}^{\prime}\left(\beta_{m}\left(\tau_{k}\right)\right)\right) p_{m+1}\left(\tau_{k}\right) \\
& +\mu_{1}\left\|p_{m}\left(\tau_{k}\right)\right\|^{2}+\mu_{2}\left\|q_{m}\left(\tau_{k}\right)\right\|^{2}, \\
q_{m+1}\left(\tau_{k}+0\right) \leq & \left(I_{k}^{\prime}\left(\alpha_{m}\left(\tau_{k}\right)\right)+G_{k}^{\prime}\left(\beta_{m}\left(\tau_{k}\right)\right)\right) q_{m+1}\left(\tau_{k}\right) \\
& +\widetilde{\mu_{1}}\left\|p_{m}\left(\tau_{k}\right)\right\|^{2}+\widetilde{\mu}_{2}\left\|q_{m}\left(\tau_{k}\right)\right\|^{2},
\end{aligned}
$$

where $\mu_{i}, \tilde{\mu_{i}}, i=1,2$ are constant vectors.

The differential inequalities (3.74)-(3.77) and the periodic conditions for the functions $p(t)$ and $q(t)$ can be written in the form

$$
\begin{gathered}
r_{m+1}^{\prime} \leq A(t) r_{m+1}+P\left\|r_{m}\right\|^{2}, \quad t \neq \tau_{k}, \\
r_{m+1}\left(\tau_{k}+0\right) \leq B_{k} r_{m+1}\left(\tau_{k}\right)+Q_{k}\left\|r_{m}\left(\tau_{k}\right)\right\|^{2}, \\
r_{m+1}(0)=r_{m+1}(T),
\end{gathered}
$$

where

$$
\begin{gathered}
r_{m+1}=\left(\begin{array}{c}
p_{m+1} \\
q_{m+1}
\end{array}\right), \quad P=\left(\begin{array}{c}
\tilde{s}+\tilde{m} \\
\tilde{s}+\tilde{m}
\end{array}\right), \\
A(t)=\left(\begin{array}{cc}
f_{x}\left(t, \alpha_{m}\right)+g_{x}\left(t, \beta_{m}\right) & 0 \\
0 & f_{x}\left(t, \alpha_{m}\right)+g_{x}\left(t, \beta_{m}\right)
\end{array}\right), \\
B_{k}=\left(\begin{array}{cc}
I_{k}^{\prime}\left(\alpha_{m}\left(\tau_{k}\right)\right)+G_{k}^{\prime}\left(\beta_{m}\left(\tau_{k}\right)\right) & 0 \\
0 & I_{k}^{\prime}\left(\alpha_{m}\left(\tau_{k}\right)\right)+G_{k}^{\prime}\left(\beta_{m}\left(\tau_{k}\right)\right)
\end{array}\right), \quad Q_{k}=\left(\begin{array}{l}
\mu_{1}+\mu_{2} \\
\widetilde{\mu_{1}}+\widetilde{\mu_{2}}
\end{array}\right) .
\end{gathered}
$$


According to Lemma 2.10, from the inequalities (3.78)-(3.80), the inequality

$$
r_{m+1}(t) \leq W(t, 0) x_{0}+\int_{0}^{t} W(t, s) P\left\|r_{m}\right\|^{2} d s+\sum_{0<t_{k}<t} W\left(t, t_{k}+\right) Q_{k}\left\|r_{m}\left(\tau_{k}\right)\right\|^{2}
$$

holds for $t \in[0, T]$, where

$$
x_{0}=(E-W(T, 0))^{-1}\left(\int_{0}^{T} W(T, s) P\left\|r_{m}\right\|^{2} d s+\sum_{k=1}^{p} W\left(T, t_{k}+\right) Q_{k}\left\|r_{m}\left(\tau_{k}\right)\right\|^{2}\right)
$$

and $W(t, s)$ is defined by $(2.17)$.

From the inequalities (3.79), (3.83), it follows that there exists a number $\lambda>0$ such that $\left\||| r_{m+1}\left|\left\|\leq\left.\lambda\left|\| r_{m}\right|\right|^{2}\right.\right.\right.$, where $\left|\left\|r|| \mid=\sup _{t \in[0, T]}\right\| r(t) \|\right.$. This inequality proves the quadratic convergence.

As particular cases of the proved theorems, we can obtain some results for the PBVP for systems of nonlinear ordinary differential equations.

Consider the PBVP for the system of ordinary differential equations

$$
\begin{gathered}
x^{\prime}=f(t, x(t))+g(t, x(t)) \quad \text { for } t \in[0, T], \\
x(0)=x(T),
\end{gathered}
$$

where $x \in \mathbb{R}^{n}, f, g:[0, T] \times \mathbb{R}^{n} \rightarrow \mathbb{R}^{n}$.

We note that PBVP (3.84) can be considered as special cases of PBVP (2.1)-(2.2) when $I_{k}=E, G_{k}=E$ for $k=1,2, \ldots, p$.

Let $\alpha, \beta \in C\left([0, T], \mathbb{R}^{n}\right)$ be such that $\alpha(t) \leq \beta(t)$. Consider the set

$$
C S(\alpha, \beta)=\left\{u \in C\left([0, T], \mathbb{R}^{n}\right): \alpha(t) \leq u(t) \leq \beta(t) \text { for } t \in[0, T]\right\} .
$$

From Theorems 3.1, 3.2, and 3.3, we obtain the following special cases for the system of periodic boundary value problem (3.84).

Theorem 3.4. Let the following conditions hold.

(1) The functions $\alpha_{0}(t), \beta_{0}(t) \in C\left([0, T], \mathbb{R}^{n}\right), \alpha_{0}(t) \leq \beta_{0}(t)$ for $t \in[0, T]$, and

$$
\begin{gathered}
\alpha_{0}^{\prime}(t) \leq f\left(t, \alpha_{0}(t)\right)+g\left(t, \beta_{0}(t)\right), \\
\beta_{0}^{\prime} \geq f\left(t, \beta_{0}(t)\right)+g\left(t, \alpha_{0}(t)\right) \quad \text { for } t \in[0, T], \\
\alpha_{0}(0) \leq \alpha_{0}(T), \quad \beta_{0}(0) \geq \beta_{0}(T) .
\end{gathered}
$$

(2) The functions $f_{x}, g_{x}$ exist and are continuous on $\Omega\left(\alpha_{0}, \beta_{0}\right), f_{x}(t, x)$ is nondecreasing in $x, g_{x}(t, x)$ is nonincreasing in $x$ for $t \in[0, T], f_{x}\left(t, \beta_{0}(t)\right) \leq 0$, and for $x \geq y$,

$$
f_{x}(t, x)-f_{x}(t, y) \leq S_{1}\|x-y\|, \quad g_{x}(t, y)-g_{x}(t, x) \leq S_{2}\|x-y\|,
$$

where $S_{1}>0, S_{2}>0$ are constant matrices, $\|\cdot\|$ is a norm in $\mathbb{R}^{n}$. 
24 Method impulsive systems with PB conditions

(3) The function $f_{x}\left(t, \beta_{0}(t)\right) x$ is quasimonotone nondecreasing in $x$ and the function $\left(f_{x}\left(t, \beta_{0}(t)\right)-g_{x}\left(t, \beta_{0}(t)\right)\right) e @ x$ is strictly decreasing in $x$ on $[0, T]$.

Then there exist two sequences of functions $\left\{\alpha_{m}(t)\right\}_{0}^{\infty}$ and $\left\{\beta_{m}(t)\right\}_{0}^{\infty}$ such that

(a) the sequences are increasing and decreasing correspondingly;

(b) both sequences uniformly converge on the intervals $\left(\tau_{k}, \tau_{k+1}\right]$ to the unique solution of the PBVP (3.84) in CS $\left(\alpha_{0}, \beta_{0}\right), k=0,1,2, \ldots, p$;

(c) the convergence is quadratic.

Theorem 3.5. Let the following conditions hold.

(1) The functions $\alpha_{0}(t), \beta_{0}(t) \in C\left([0, T], \mathbb{R}^{n}\right), \alpha_{0}(t) \leq \beta_{0}(t)$ for $t \in[0, T]$, and

$$
\begin{gathered}
\alpha_{0}^{\prime}(t) \leq f\left(t, \beta_{0}(t)\right)+g\left(t, \alpha_{0}(t)\right), \\
\beta_{0}^{\prime}(t) \geq f\left(t, \alpha_{0}(t)\right)+g\left(t, \beta_{0}(t)\right) \quad \text { for } t \in[0, T], \\
\alpha_{0}(0) \leq \alpha_{0}(T), \quad \beta_{0}(0) \geq \beta_{0}(T) .
\end{gathered}
$$

(2) The functions $f_{x}, g_{x}$ exist and are continuous on $\Omega\left(\alpha_{0}, \beta_{0}\right), f_{x}(t, x)$ is nondecreasing in $x, g_{x}(t, x)$ is nonincreasing in $x$ for $t \in[0, T], f_{x}\left(t, \beta_{0}(t)\right) \leq 0$, and for $x \geq y$,

$$
f_{x}(t, x)-f_{x}(t, y) \leq S_{1}\|x-y\|, \quad g_{x}(t, y)-g_{x}(t, x) \leq S_{2}\|x-y\|,
$$

where $S_{1}>0, S_{2}>0$ are constant matrices, $\|\cdot\|$ is a norm in $\mathbb{R}^{n}$.

(3) The function $g_{x}\left(t, \beta_{0}(t)\right) x$ is quasimonotone nondecreasing in $x$ and the function $\left(-f_{x}\left(t, \alpha_{0}\right)+g_{x}\left(t, \alpha_{0}\right)\right) e @ x$ is strictly decreasing in $x$ on $[0, T]$.

Then there exist two sequences of functions $\left\{\alpha_{m}(t)\right\}_{0}^{\infty}$ and $\left\{\beta_{m}(t)\right\}_{0}^{\infty}$ such that

(a) the sequences are increasing and decreasing correspondingly;

(b) both sequences uniformly converge on the intervals $\left(\tau_{k}, \tau_{k+1}\right]$ to the unique solution of the PBVP (3.84) in CS $\left(\alpha_{0}, \beta_{0}\right), k=0,1,2, \ldots, p$;

(c) the convergence is quadratic.

Theorem 3.6. Let the following conditions hold.

(1) The functions $\alpha_{0}(t), \beta_{0}(t) \in C\left([0, T], \mathbb{R}^{n}\right), \alpha_{0}(t) \leq \beta_{0}(t)$ for $t \in[0, T]$, are such that

$$
\begin{gathered}
\alpha_{0}^{\prime}(t) \leq f\left(t, \alpha_{0}(t)\right)+g\left(t, \alpha_{0}(t)\right), \\
\beta_{0}^{\prime}(t) \geq f\left(t, \beta_{0}(t)\right)+g\left(t, \beta_{0}(t)\right) \quad \text { for } t \in[0, T], \\
\alpha_{0}(0) \leq \alpha_{0}(T), \quad \beta_{0}(0) \geq \beta_{0}(T) .
\end{gathered}
$$

(2) The functions $f_{x}, g_{x}$ exist and are continuous on $\Omega\left(\alpha_{0}, \beta_{0}\right), f_{x}(t, x)$ is nondecreasing in $x, g_{x}(t, x)$ is nonincreasing in $x$ for $t \in[0, T]$, and for $x \geq y$,

$$
f_{x}(t, x)-f_{x}(t, y) \leq S_{1}\|x-y\|, \quad g_{x}(t, y)-g_{x}(t, x) \leq S_{2}\|x-y\|,
$$

where $S_{1}>0, S_{2}>0$ are constant matrices, $\|\cdot\|$ is a norm in $\mathbb{R}^{n}$.

(3) The function $\left(f_{x}\left(t, \alpha_{0}(t)\right)+g_{x}\left(t, \beta_{0}(t)\right)\right) x$ is quasimonotone nondecreasing in $x$ and the function $\left(f_{x}\left(t, \beta_{0}\right)+g_{x}\left(t, \alpha_{0}\right)\right) e @ x$ is strictly decreasing in $x$ on $[0, T]$. 
Then there exist two sequences of functions $\left\{\alpha_{m}(t)\right\}_{0}^{\infty}$ and $\left\{\beta_{m}(t)\right\}_{0}^{\infty}$ such that

(a) the sequences are increasing and decreasing correspondingly;

(b) both sequences uniformly converge on the intervals $\left(\tau_{k}, \tau_{k+1}\right]$ to the unique solution of the PBVP (3.84) in CS $\left(\alpha_{0}, \beta_{0}\right), k=0,1,2, \ldots, p$;

(c) the convergence is quadratic.

\section{References}

[1] D. D. Baĭnov and S. G. Hristova, The method of quasilinearization for the periodic boundary value problem for systems of impulsive differential equations, Applied Mathematics and Computation 117 (2001), no. 1, 73-85.

[2] R. E. Bellman and R. E. Kalaba, Quasilinearization and Nonlinear Boundary-Value Problems, Modern Analytic and Computional Methods in Science and Mathematics, vol. 3, American Elsevier, New York, 1965.

[3] S. G. Hristova and G. K. Kulev, Quasilinearization of a boundary value problem for impulsive differential equations, Journal of Computational and Applied Mathematics 132 (2001), no. 2, 399-407.

[4] V. Lakshmikantham, An extension of the method of quasilinearization, Journal of Optimization Theory and Applications 82 (1994), no. 2, 315-321.

[5] V. Lakshmikantham, D. D. Bănov, and P. S. Simeonov, Theory of Impulsive Differential Equations, Series in Modern Applied Mathematics, vol. 6, World Scientific, New Jersey, 1989.

[6] V. Lakshmikantham and J. J. Nieto, Generalized quasilinearization for nonlinear first order ordinary differential equations, Nonlinear Times and Digest 2 (1995), no. 1, 1-9.

[7] V. Lakshmikantham, N. Shahzad, and J. J. Nieto, Methods of generalized quasilinearization for periodic boundary value problems, Nonlinear Analysis. Theory, Methods \& Applications. Series A: Theory and Methods 27 (1996), no. 2, 143-151.

[8] V. Lakshmikantham and A. S. Vatsala, Generalized Quasilinearization for Nonlinear Problems, Mathematics and Its Applications, vol. 440, Kluwer Academic, Dordrecht, 1998.

[9] A. M. Samoulenko and N. A. Perestyuk, Impulsive Differential Equations, World Scientific Series on Nonlinear Science. Series A: Monographs and Treatises, vol. 14, World Scientific, New Jersey, 1995.

S. G. Hristova: Department of Mathematics and Computer Science, Denison University, Granville, Ohio, OH 43023, USA

E-mail address: snehri@pu.acad.bg

A. S. Vatsala: Department of Mathematics, University of Louisiana, Lafayette, LA 70504, USA

E-mail address: vatsala@louisiana.edu 


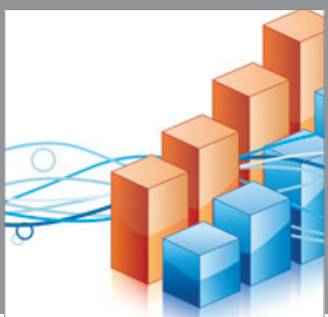

Advances in

Operations Research

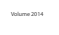

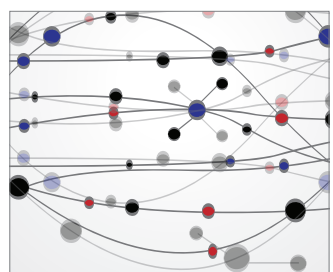

\section{The Scientific} World Journal
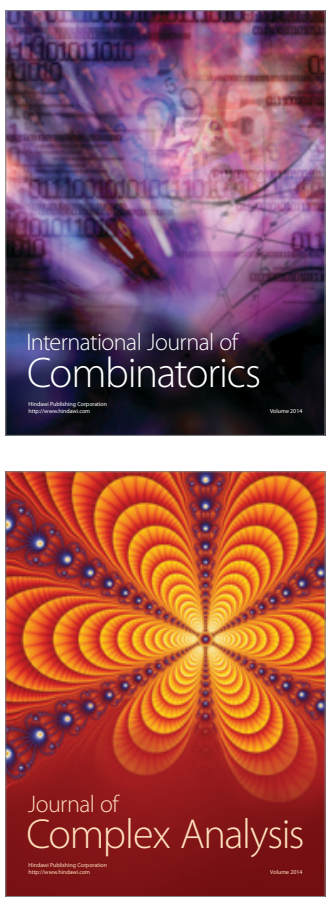

International Journal of

Mathematics and

Mathematical

Sciences
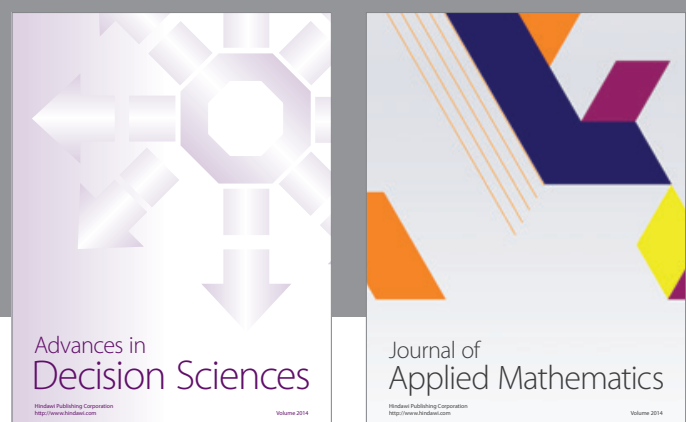

Journal of

Applied Mathematics
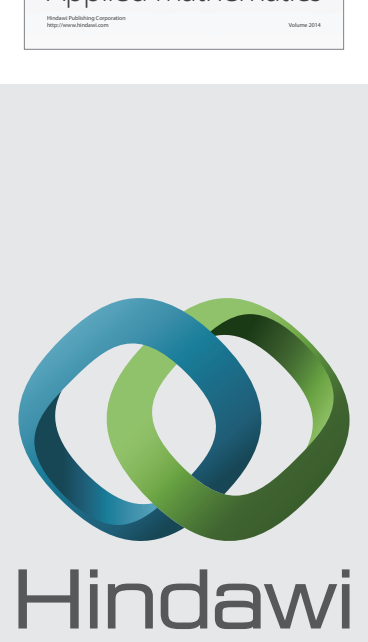

Submit your manuscripts at http://www.hindawi.com
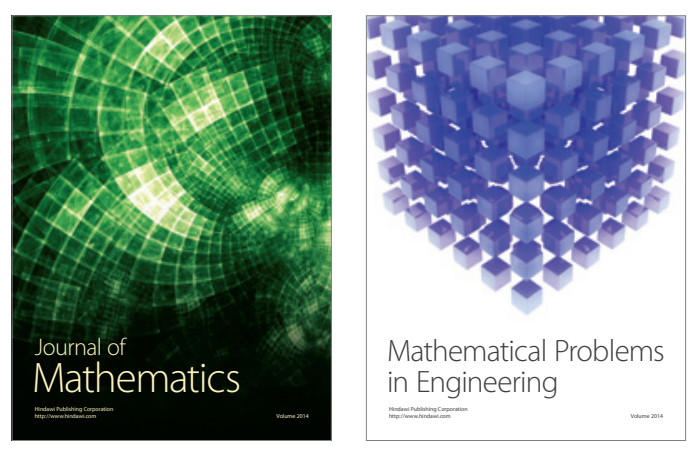

Mathematical Problems in Engineering
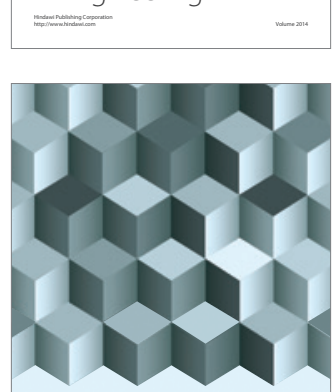

Journal of

Function Spaces
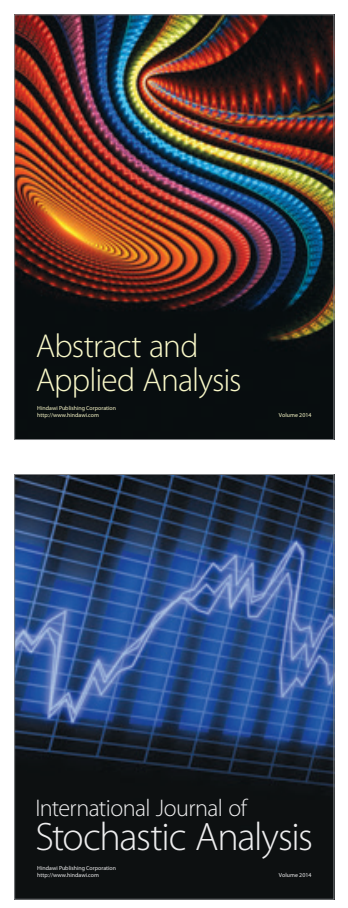

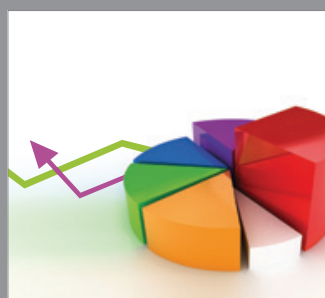

ournal of

Probability and Statistics

Promensencen
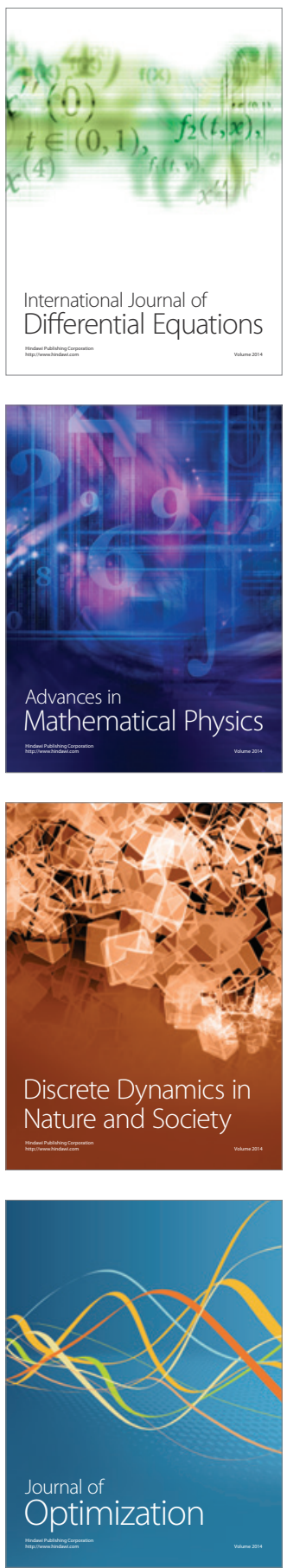